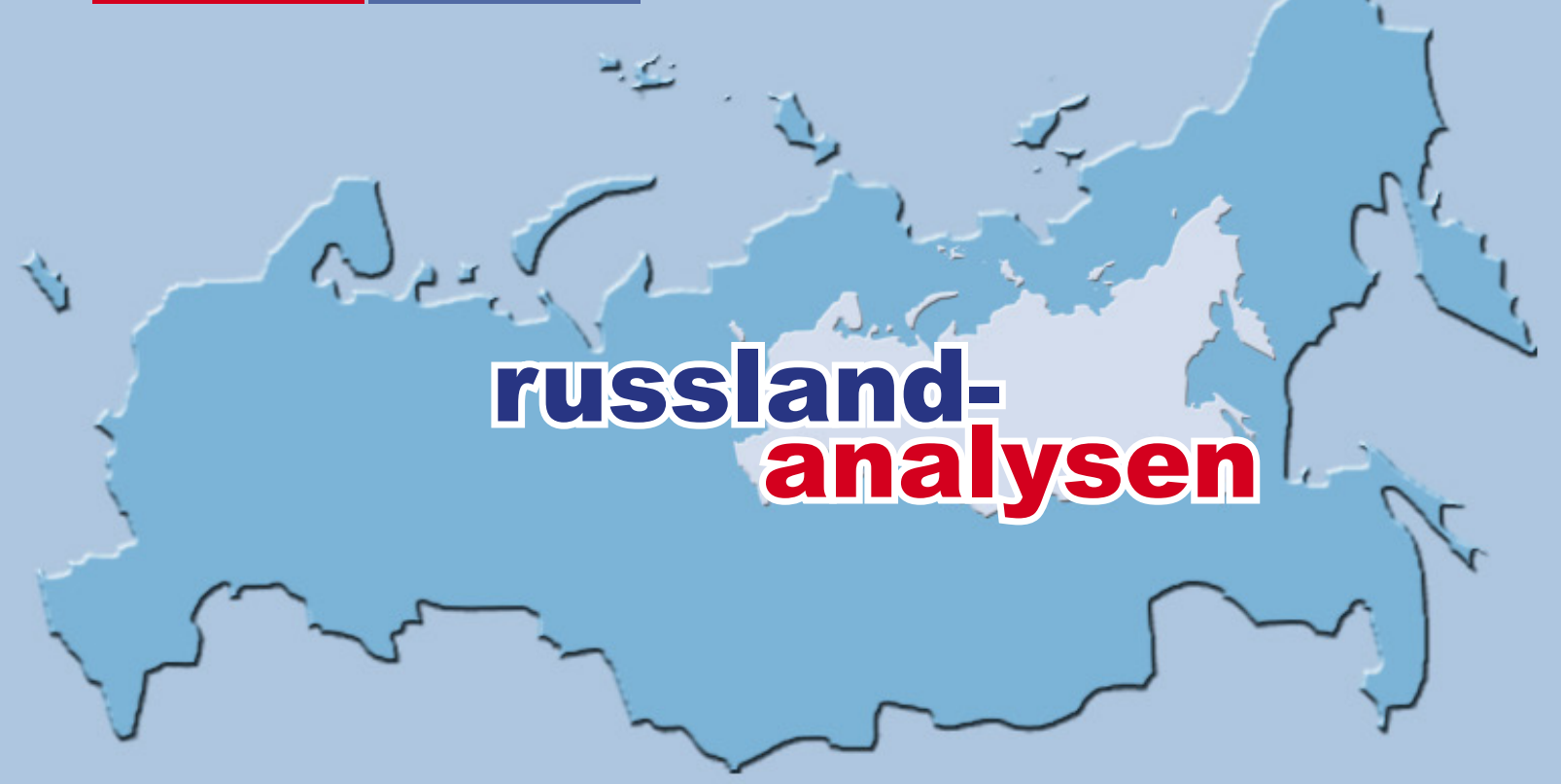

http://www.laender-analysen.de/russland/

\title{
REGIONALE INTEGRATION IN EURASIEN
}

VON DER REDAKTION

Einführung

ANALYSE

Regionale wirtschaftliche Integration in Eurasien: Die Eurasische Wirtschaftsunion

Alexander Libman (Ludwig-Maximilians-Universität München)

- STATISTIK

Die Eurasische Wirtschaftsunion in Zahlen

UMFRAGE

Einstellungen zur Eurasischen Wirtschaftsunion

aNALYSE

Das System kollektiver Sicherheit in Eurasien

Julija Nikitina (Moskauer Staatliche Hochschule für internationale Beziehungen)

STATISTIK

Militärausgaben der OVKS-Mitgliedsstaaten

UMFRAGE

Einstellung der russischen Bevölkerung zum Fall Skripal

\section{CHRONIK}

3. - 14. April 2018

\section{DEUTSCHES \\ POIFN \\ INSTITUT}

Forschungsstelle Osteuropa an der Universität Bremen

Leibniz-Institut für

Agrarentwicklung in Transformationsökonomien

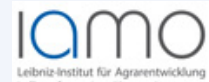

Leibniz-Institut für st- und Südosteuropaforschung
Zentrum für Osteuropa- und internationale Studien (ZOiS) $\mathrm{gGmbH}$ 


\section{Einführung}

Das Leibniz-Institut für Ost- und Südosteuropaforschung (IOS) freut sich, Teil des neukonstituierten Publikationskonsortiums der Länder-Analysen zu sein und das Redaktionsteam ergänzen zu dürfen. Die erste vom IOS maßgeblich mitgestaltete Ausgabe widmet sich regionalen Organisationen in Eurasien und nimmt zwei Bereiche in den Blick: die wirtschaftliche Integration am Beispiel der Eurasischen Wirtschaftsunion (EAWU) und die institutionalisierte politisch-militärische Kooperation am Beispiel der Organisation des Vertrags über kollektive Sicherheit (OVKS). Beide Organisationen werden zumeist als ineffektiv und schlichtweg irrelevant und/oder als rein machtpolitisches Projekt Russlands angesehen. Mit diesen vermeintlich selbstverständlichen Annahmen und Wertungen setzen sich die Beiträge in dieser Ausgabe kritisch auseinander.

Alexander Libman stellt in seinem Beitrag zur EAWU fest, dass gerade für Russland die unmittelbaren Mitgliedschaftsvorteile alles andere als einleuchtend sind. Zudem haben die kollektiven Organe der Organisation bereits Entscheidungen getroffen, die der russischen Position zuwiderliefen (siehe dazu auch den Beitrag von Ann-Sophie Gast zu Kirgistans Mitgliedschaft in der Eurasischen Wirtschaftsunion in der aktuellen Ausgabe der Zentralasien-Analysen vom 29. März 2018). Die EAWU verfügt somit durchaus über Funktionalität und eine selbstständige institutionelle Struktur. Obwohl die EAWU als Regionalorganisation im Bereich der wirtschaftlichen Integration mehr als ihre Vorgängerinnen erreicht hat, scheint Russland jedoch angesichts der Konflikte in der Ukraine und Syrien sowie der Spannungen in seinen Beziehungen zu den USA und zur EU langsam sein Interesse an diesem Integrationsprojekt zu verlieren.

Julija Nikitina konstatiert in ihrem Beitrag, dass die OVKS unter einer mangelnden Balance zwischen den militärischen Fähigkeiten ihrer Mitgliedsstaaten leidet. Ein Fakt, der die militärische Integration in die OVKS für Russland eher unattraktiv macht. Darüber hinaus fehlt es der Organisation an klaren gemeinsamen Werten und Zielen. Dennoch wäre es nicht angebracht, die OVKS als nutzlose Organisation abzuschreiben: Über die Jahre entstand institutionelles Wissen über Terrorabwehr, welches für die transregionale Kooperation, zum Beispiel im Rahmen der OSZE, nützlich sein könnte.

Beide Beiträge lassen das Fazit zu, dass in der Praxis sowohl die EAWU als auch die OVKS vor allem im Bereich der praktischen Kooperation auf den Arbeitsebenen derzeit ihr Potential und ihre Stärken entfalten.

Über den thematischen Fokus auf die regionale Integration in Eurasien hinaus stellen wir unseren Leserinnen und Lesern mit den Ergebnissen aktueller Umfragen zur öffentlichen Wahrnehmung des Falls Skripal in Russland nützliche Informationen und Perspektiven zu einem tagespolitisch relevanten Thema vor.

Wir wünschen Ihnen eine angenehme und informative Lektüre!

Evgeniya Bakalova (IOS)

zuständige Redakteurin 


\title{
Regionale wirtschaftliche Integration in Eurasien: Die Eurasische Wirtschaftsunion
}

\author{
Alexander Libman (Ludwig-Maximilians-Universität München)
}

\section{Zusammenfassung}

Die Eurasische Wirtschaftsunion (EAWU) ist die erste regionale Wirtschaftsorganisation in Eurasien, die sich nicht lediglich auf die Integrationsrhetorik beschränkt. Die EAWU ist eine funktionsfähige Zollunion und zeigt gewisse Fortschritte auch in anderen Integrationsbereichen. Jedoch ist die EAWU - trotz des Namens - keineswegs als Pendant zur EU zu verstehen. Aber auch die Sicht, dass die EAWU lediglich ein Machtinstrument Russlands sei, wäre eine massive Vereinfachung. Für die Zukunft ist davon auszugehen, dass die EAWU ihre Funktionalität behält. Allerdings ist ein weiterer starker Fortschritt der Integration unwahrscheinlich, auch deswegen, weil Russland langsam sein Interesse an der EAWU zu verlieren scheint.

\section{Wirtschaftliche Verflechtungen und politische Asymmetrien}

Wirtschaftliche Fragen wurden sehr früh zu einem Kernstück der eurasischen regionalen Integration, weil die wirtschaftlichen Reformen in den 1990er Jahren als die Kernaufgabe der neuen unabhängigen Staaten empfunden wurden. Ganz wie im Sicherheitsbereich ist die Entwicklung der eurasischen wirtschaftlichen Integration durch zwei Merkmale geprägt, nämlich durch die sowjetische Vergangenheit und die bestehenden Machtasymmetrien. Der Zerfall der UdSSR hinterließ Volkswirtschaften der Länder Eurasiens, die stark miteinander verflochten waren: durch die gemeinsame Infrastruktur im Transport- und Energiebereich; durch die Abhängigkeit von Rohstofflieferungen aus anderen Ländern der Region und durch die technologischen Komplementaritäten im verarbeitenden Gewerbe. Das wichtigste Erbe der sowjetischen Zeit ist jedoch die Verbreitung der russischen Sprache und die Gemeinsamkeiten im Verhalten und in der Kultur. Sie wurden zu einer der Grundlagen der neuen, marktwirtschaftlichen Verflechtungen, die im Zuge der Transformation entstanden sind, etwa durch Direktinvestitionen oder Arbeitsmigration.

Interessanterweise führen die wirtschaftlichen Verflechtungen in Eurasien nicht automatisch zu einem Erfolg der wirtschaftlichen Integration (wie am Beispiel der Integrationsprojekte der 1990er Jahre zu sehen ist). Es ist eher umgekehrt: Für viele Länder sind gerade die starken wirtschaftlichen Abhängigkeiten von anderen Staaten Eurasiens, insbesondere Russland, ein Grund zur Sorge. Das liegt an einer zweiten Besonderheit der Region, nämlich der enormen wirtschaftlichen Asymmetrie zugunsten Russlands. Ohne die Russische Föderation wären die Verbindungen zwischen den einzelnen Staaten der Region zu vernachlässigen. Selbst in der Subregion Zentralasien spielen die Beziehungen der Länder zueinander eine geringere Rolle als jene zu Russland. Dabei scheut Russland nicht davor zurück, diese
Abhängigkeit als Druckmittel zu verwenden. Bereits in den 1990er Jahren hatte Russland regelmäßig formale und informelle Wirtschaftssanktionen gegen seine Nachbarländer eingesetzt. In den 2000er Jahren wurde dieser Ansatz noch stärker verfolgt. Deswegen sollte es nicht verwundern, dass die tatsächlichen Erfolge der regionalen Integration in Eurasien gerade in einer Zeit erreicht wurden, als die wirtschaftlichen Verflechtungen zwischen den beiden für die eurasische Integration wichtigsten Staaten - Kasachstan und Russland im Vergleich zu den 1990er Jahren deutlich abnahmen.

\section{Eurasische Wirtschaftsunion}

Bis zum Jahr 2000 blieb die regionale Integration in Eurasien primär eine Frage der Rhetorik, die von den Regierungen gern eingesetzt wurde, um innenpolitisch ihre Popularität zu steigern. Das blieb jedoch fast ohne tatsächliche wirtschaftspolitische Konsequenzen. Die Situation änderte sich im Jahr 2010 mit der Gründung einer Zollunion von Kasachstan, Belarus und Russland. 2015 wurde aus der Zollunion die Eurasische Wirtschaftsunion; im selben Jahr traten der Organisation mit Armenien und Kirgistan zwei weitere Länder bei. Kernstück der EAWU bleibt die Handelsintegration, die auf drei Grundsätzen fußt: Abschaffung der internen Zölle und Handelsgrenzen, Harmonisierung der Zölle gegenüber Drittändern und Übertragung der handelspolitischen Kompetenzen an die Eurasische Wirtschaftskommission. Hinzu kommen die Freizügigkeit der Menschen und des Kapitals.

Die EAWU ist eindeutig kein Pendant zur EU und wird es auch nicht werden. Formal scheint die EU tatsächlich das Muster zu sein, nach dem die EAWU aufgebaut wurde. Inhaltlich ist die Funktionsweise der EAWU-Institutionen ganz anders als die der EU und der Grad der Kooperation in der EAWU viel bescheidener. Jedoch ist das bereits erreichte Niveau der Integration in der EAWU im internationalen Vergleich nicht zu unterschätzen: Es gibt nur sehr wenige funktionsfä- 
hige Zollunionen in der Welt. Bis zum Jahr 2015 wurden mehr als $90 \%$ der Zölle von den Regularien der EAWU erfasst. Nach dem Beitritt Kasachstans zur WTO hat das Land unilateral beschlossen, Zölle für mehrere Güter zu senken, weswegen hier zurzeit nur ca. 60 \% der Zollsätze harmonisiert sind. Eine sehr große Lücke in den Regeln der EAWU stellen die sogenannten nichttarifären Handelshemmnisse dar, beispielsweise Güterzertifizierungen oder Gesundheitsvorschriften. Sie verbleiben im Entscheidungsbereich der einzelnen Staaten und stellen daher für den internen Handel eine wichtige Hürde dar. Die Einführung der russischen Sanktionen gegen die EU (Verbot landwirtschaftlicher Importe) ist ein weiteres Problem für die EAWU, da diese Embargos nicht von anderen EAWU-Staaten mitgetragen wurden.

Es gibt vereinzelt Fälle, in denen EAWU-Länder aus politischen Gründen die Kontrollen an den internen Grenzen der EAWU wiederherstellen. So hatte Kasachstan im Jahr 2017 für mehrere Wochen die Grenze zu Kirgistan geschlossen. Dieser Maßnahme war heftige Kritik des damaligen kirgisischen Präsidenten an die Adresse Kasachstans wegen einer angeblichen kasachischen Einmischung in die kirgisischen Wahlen vorausgegangen. Im Jahr 2014 hat Russland ähnliche Maßnahmen gegen Belarus ergriffen, weil Belarus intensiv dazu genutzt wurde, das russische Lebensmittelembargo gegen die EU zu umgehen. Beide Konflikte wurden zwar nach einigen Monaten gelöst, beeinträchtigen aber die Glaubwürdigkeit der EAWU.

Neben der EAWU bestehen in Eurasien noch zwei weitere wichtige Institutionen der wirtschaftlichen Integration, die allerdings eng mit der EAWU verbunden sind. Die Eurasische Entwicklungsbank konzentriert sich auf die Vergabe von Krediten zur Förderung der EAWUVolkswirtschaften und der regionalen Integration. Der Eurasische Stabilisierungs- und Entwicklungsfonds wurde im Zuge der Krise von 2008 gegründet, um die schwächeren Volkswirtschaften der Region zu stützen. Heute finanziert er auch langfristige Strukturprojekte in der Region.

\section{EAWU - politisch, russlandzentriert, irrelevant?}

In der Diskussion über die EAWU werden von den Wissenschaftlern und Experten regelmäßig drei Feststellungen als selbstverständlich angenommen. Erstens sei die EAWU primär ein politisches Projekt und die wirtschaftliche Zielsetzung und Wirksamkeit der Organisation sei zu vernachlässigen. Zweitens sei die EAWU eindeutig von Russland dominiert und die kleineren Staaten hätten in der Union keinen Einfluss. Drittens sei die EAWU schlichtweg irrelevant: Die Organisation sei unfähig, selbständige Entscheidungen zu treffen (die EAWU verfüge also nicht über Actorness, wie es in der politikwissenschaftlichen Diskussion genannt wird).
Alle drei Stellungnahmen sind keineswegs unstrittig. Betrachtet man die Dokumente und formalen Erklärungen, so ist die EAWU als Institution eindeutig nicht politisch: Es fehlen selbst rudimentäre Bekenntnisse zu politischen Werten oder symbolische gemeinsame politische Institutionen, was auf die eindeutige Haltung Kasachstans hierzu zurückzuführen ist. Die Situation stellt sich jedoch komplizierter dar, wenn man die Deutung der EAWU durch einzelne Mitgliedsstaaten betrachtet, was im Folgenden geschehen soll. Darüber hinaus ist es auch nicht zutreffend, dass die EAWU-Staaten keine wirtschaftlichen Gewinne aus deren Mitgliedschaft erzielen können. Für Kirgistan und Armenien bedeutet die Mitgliedschaft in der EAWU durch die Umverteilung der gemeinsamen Zolleinnahmen höhere Haushaltseinnahmen und einen freien Zugang von Migranten zum russischen Arbeitsmarkt. Belarus profitiert von erheblichen russischen Subventionen (insbesondere durch reduzierte Rohstoffpreise). Interessanterweise ist die Frage am schwierigsten zu klären, inwieweit Russland von der EAWU wirtschaftlich profitiert.

Die Institutionen der EAWU als eindeutig von Russland dominiert zu bezeichnen, erscheint ebenfalls als eine Vereinfachung. Den formal bestehenden Regeln zufolge werden die Entscheidungen in der EAWU entweder auf Konsensbasis oder durch die einfache Stimmenmehrheit der Vertreter der Mitgliedsstaaten gemacht. Die Eurasische Wirtschaftskommission hat bereits Entscheidungen getroffen, die entweder der russischen Position zuwiderliefen oder sogar russische Behörden dazu auffordern, nationale Verordnungen und Regulierungen zu ändern. Zur Illustration ein Beispiel: Im Jahr 2014 scheiterte Russland mit dem Versuch, zwei andere Mitglieder der Zollunion zu überzeugen, Freihandelsabkommen mit der Ukraine aufzukündigen. Russland hat das zwar anderthalb Jahre später im Alleingang vollzogen, doch zeigt der Fall deutlich, dass die EAWU nicht immer das beschließt, was von Russland gewollt ist.

Hinzu kommt, dass die EAWU kein Produkt russischen Drucks auf andere Mitgliedsstaaten ist. Lediglich Armenien scheint der EAWU nach dem expliziten Druck Russlands und in der Befürchtung, russische Sicherheitsgarantien zu verlieren, beigetreten zu sein. Kasachstan hingegen hat die EAWU mitinitiiert und massiv unterstützt. Ohne die proaktive Haltung dieses Staates wäre die EAWU nicht zustande gekommen.

Was die Actorness der EAWU angeht, so ist die Situation ebenfalls nicht eindeutig. Die Forschung zu diesem Thema ist allerdings alles andere als umfangreich. Es ist jedoch festzustellen, dass die EAWU sich - anders als die EU - in ihren Beziehungen zu den Mitgliedsstaaten nicht als unabhängiger Akteur versteht. Das ist nicht verwunderlich, da die meisten Mitglieder der EAWU Auto- 
kratien sind, die eine solche Reduzierung ihrer Macht nicht tolerieren würden. Zum Teil wurden die Institutionen der EAWU, die ursprünglich größere Möglichkeiten hatten, sich von den Mitgliedsstaaten zu emanzipieren, konsequent durch institutionelle Reformen geschwächt. Ein Beispiel hierfür ist das Gericht der EAWU. Noch wichtiger ist, dass die Bürokraten in den Apparaten der EAWU, die um ihre Karrieren zu fürchten haben, es in vielen Fällen bevorzugen, vorsichtig zu handeln und alle möglichen Gegensätze auf die politische Ebene zu verschieben - dieselbe Haltung ist auch auf nationaler Ebene in den EAWU-Staaten zu beobachten.

Die Actorness der EAWU manifestiert sich allerdings auf eine andere Weise, nämlich nicht in ihren Beziehungen zu den Mitgliedsstaaten, sondern in den Versuchen der Eurasischen Wirtschaftskommission, in einen Wettbewerb mit einzelnen nationalen Ressorts dieser Staaten zu treten und den politischen Entscheidungsträgern ihren Nutzen gegenüber diesen Ressorts zu beweisen. Hinzu kommt, dass die EAWU - wie andere regionale Organisationen auch - ihre Actorness zudem durch die Entwicklung der Beziehungen zu anderen regionalen Organisationen stärken kann. Es geht dabei nicht nur um die eurasischen Organisationen, sondern auch um Gruppen und Projekte außerhalb der Region - ein Beispiel sind die Verhandlungen über eine »Kongruenz« zwischen der EAWU und der chinesischen »Belt and Road«-Initiative.

\section{Wie geht es weiter?}

Jahrzehnte des Scheiterns von Versuchen einer Wirtschaftsintegration in Eurasien führen zu berechtigten Zweifeln an der langfristigen Funktionalität der EAWU. Diese regionale Organisation hat jedoch bereits viel mehr erreicht, als alle ihre Vorgänger, und es gibt heute zumindest keine Belege, dass die Mitgliedsstaaten in absehbarer Zeit von ihren Verpflichtungen in der EAWU stark abweichen werden, obwohl auch weiterhin gelegentliche Abweichungen vorkommen werden. So hat etwa Kasachstan nach der Abwertung des russischen Rubel im Jahr 2014 keine Schutzmaßnahmen gegen billiger gewordene russische Importe eingeführt (1998 noch hat das Land in einer ähnlichen Situation sofort protektionistische Barrieren geschaffen). Eine Veränderung dieser Situation erscheint unwahrscheinlich.

Ebenso unwahrscheinlich ist allerdings auch eine starke Vertiefung der regionalen Integration. Die EAWU-Länder vermeiden eine zu starke Abhängigkeit voneinander und von Russland: Die letzten zwei großen Fortschritte der EAWU vom Jahr 2017 - der gemeinsame Markt für Arzneimittel und das neue Zollgesetzbuch - wurden nur in einer Kompromissfassung verabschiedet, die viele ursprünglich geplanten Integrationsschritte ausschließt.

Von noch größerer Bedeutung sind jedoch die vermehrten Anzeichen, dass Russland sein Interesse an der EAWU verliert. Wie bereits erwähnt, leuchten - schaut man auf die Funktionsweise der EAWU - gerade für Russland die Vorteile nicht sonderlich ein: Die Märkte der anderen EAWU-Staaten sind schlicht zu klein, um für Russland Gewinne zu schaffen. Für eine Erklärung, warum Russland die EAWU mitträgt, sollte man daher vielleicht nicht nur auf objektive wirtschaftliche Vorteile schauen, sondern auch die Wahrnehmungen der russischen Eliten betrachten. Als die EAWU gegründet wurde, wurde sie von der russischen Führung als ein Zeichen der politischen Bedeutung Russlands gesehen, sowohl global, als auch im eurasischen Raum. Um dieses Zeichen zu wahren, war Russland auch bereit, gegenüber anderen Ländern Eurasiens Zugeständnisse zu machen, und Moskau hat sich intensiv dafür eingesetzt, dass die EAWU international (etwa von der EU) anerkannt wird. Diese Vorstellung von der geopolitischen Bedeutung der EAWU scheint wenig mit dem eigentlichen Wesen der Organisation zu tun zu haben, prägt aber gleichwohl das russische Handeln in Bezug auf diese Organisation.

Im Laufe der Zeit ist die EAWU jedoch aus dem Fokus der russischen Führung gewichen. Die Aufmerksamkeit der politischen Führung in Moskau ist jetzt vielmehr auf die Krisen um die Ukraine oder in Syrien, auf die Kooperation mit China und anderen asiatischen Ländern oder auf die zahlreichen Krisen in den Beziehungen zu den USA und der EU gerichtet, die für den Großmachtanspruch Russlands jetzt bedeutender zu sein scheinen. In Putins jüngster Rede zur Lage der Nation im März 2018 wurde die EAWU nur am Rande erwähnt. Das bedeutet nicht, dass die EAWU von Russland nicht mehr unterstützt wird, sie scheint jetzt lediglich als nicht sehr wichtig wahrgenommen zu werden.

Das kann paradoxerweise sogar Vorteile für die EAWU bedeuten, indem sie sich noch stärker auf rein technische Handels- und Wirtschaftsfragen (wo echte Fortschritte möglich sind) konzentrieren kann. Aber es erhöht auch die Wahrscheinlichkeit, dass Russland bei seinen (wirtschafts-)politischen Entscheidungen die Interessen der EAWU-Staaten nicht mehr berücksichtigen wird. Das dürfte die Kooperation in der EAWU negativ beeinflussen.

\section{Über den Autor}

Alexander Libman ist Professor für Sozialwissenschaftliche Osteuropastudien an der Ludwig-Maximilians-Universität München. 
Lesetipps

- Dragnewa, Rilka; Kataryna Wolczuk (Hg.): Eurasian Economic Integration: Law, Policy and Politics. Cheltenham: Edward Elgar 2013.

- Libman, Alexander; Evgeny Vinokurov: Holding-Together Regionalism: Twenty Years of Post-Soviet Integration, Basingstoke: Palgrave MacMillan 2012.

- Vinokurov, Evgeny: Introduction to the Eurasian Economic Union. Basingstoke: Palgrave MacMillan 2018.

\section{Die Eurasische Wirtschaftsunion in Zahlen}

Grafik 1: Binnen- und Außenhandel der EAWU-Mitgliedsstaaten (in Mrd. US-Dollar), 2016

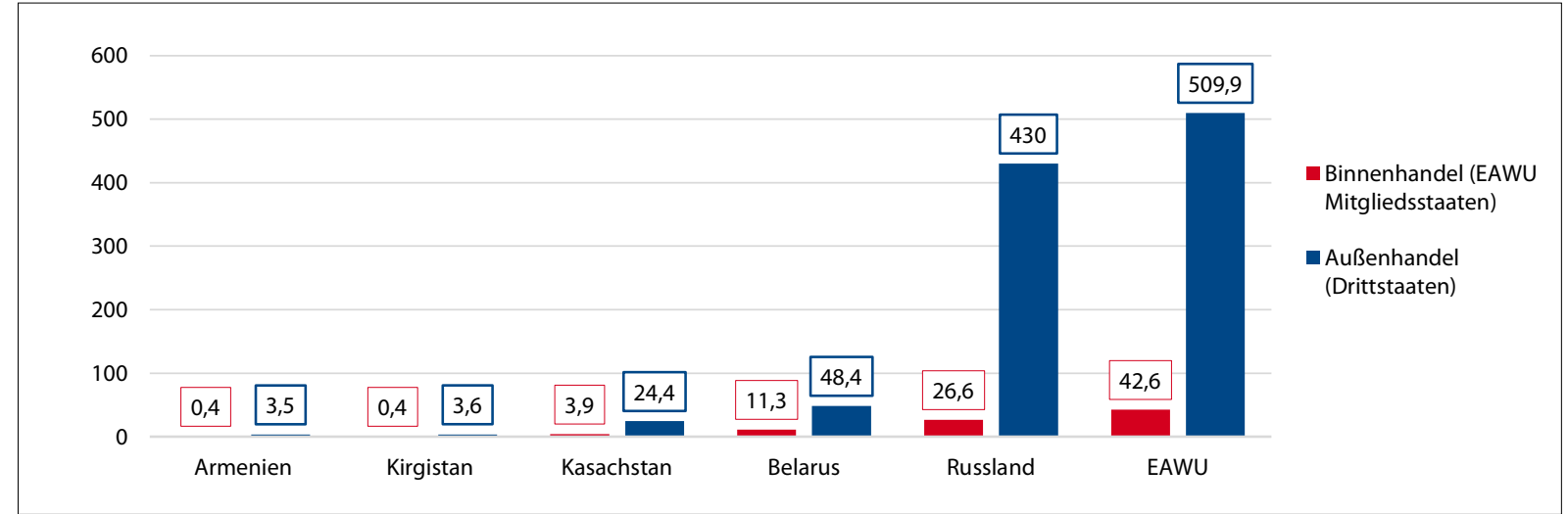

Quelle: Eurasian Development Bank, Eurasian Economic Integration, 2017, <https://eabr.org/upload/iblock/470/EDB-Centre_2017_ Report-43_EEI_ENG.pdf>, S. 9; Eurostat, Internationaler Warenverkehr, 2017, <http://ec.europa.eu/eurostat/statistics-explained/ index.php/International_trade_in_goods/de\#Extra-EU-Warenhandel>

Grafik 2: Binnen- und Außenhandel der EAWU- und der EU-Mitgliedsstaaten im Vergleich, 2016 (in Prozent)

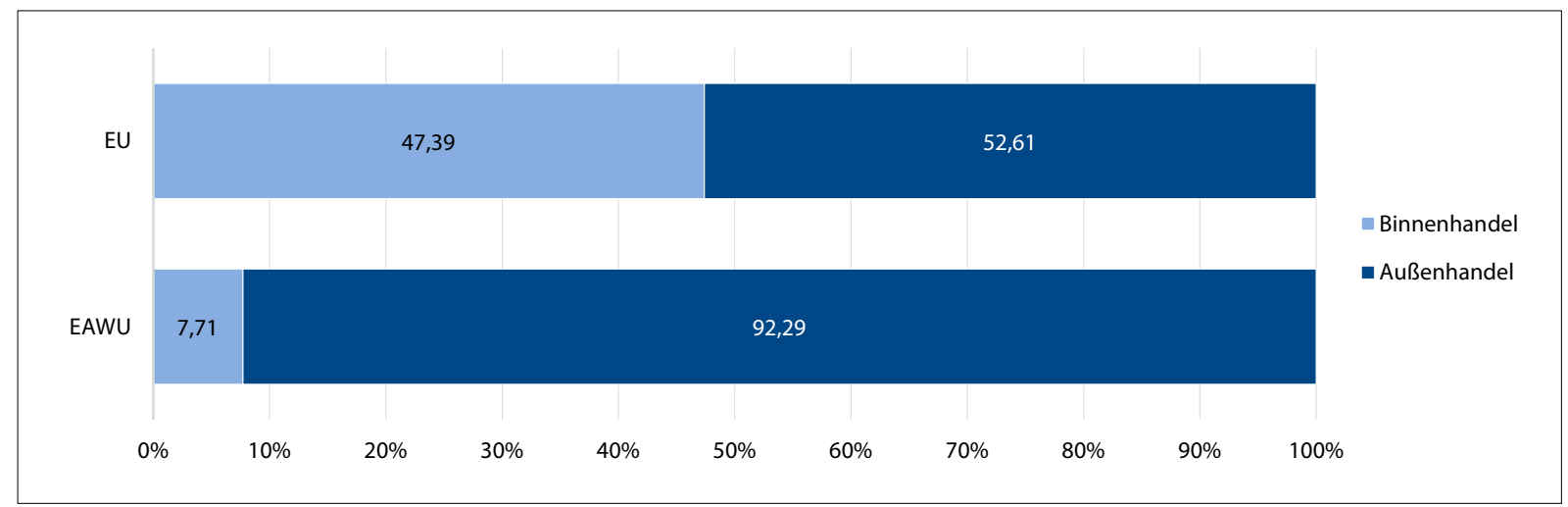

Quelle: Eurasian Development Bank, Eurasian Economic Integration, 2017, <https://eabr.org/upload/iblock/470/EDB-Centre_2017_ Report-43_EEI_ENG.pdf>, S. 9; Eurostat, Internationaler Warenverkehr, 2017, <http://ec.europa.eu/eurostat/statistics-explained/ index.php/International_trade_in_goods/de\#Extra-EU-Warenhandel> 
Grafik 3: Warenstruktur des EAWU-Binnenhandels (in Prozent), 2016

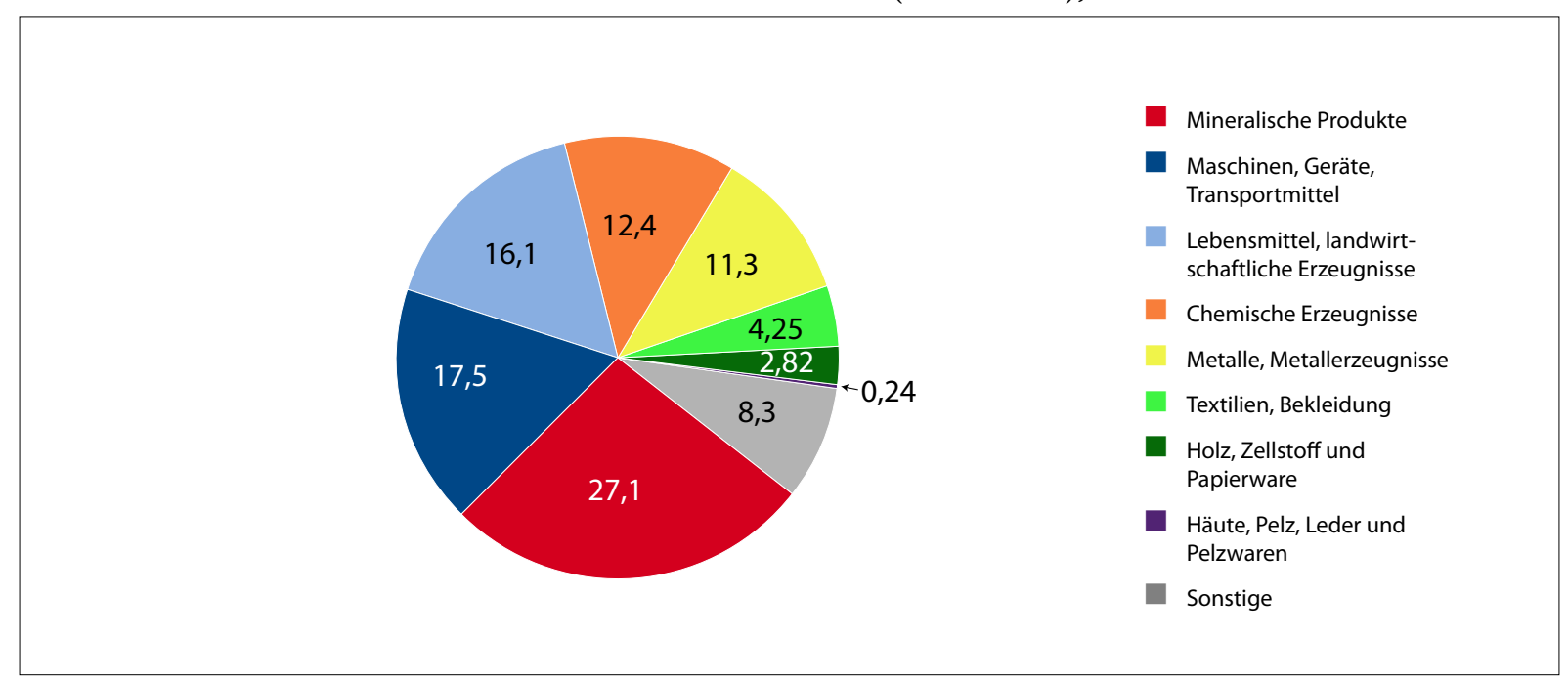

Quelle: Eurasian Development Bank, Eurasian Economic Integration, 2017, <https://eabr.org/upload/iblock/470/EDB-Centre_2017_ Report-43_EEI_ENG.pdf>, S. 53

Grafik 4: Warenstruktur des Handels mit Drittstaaten (ohne EAWU), 2016

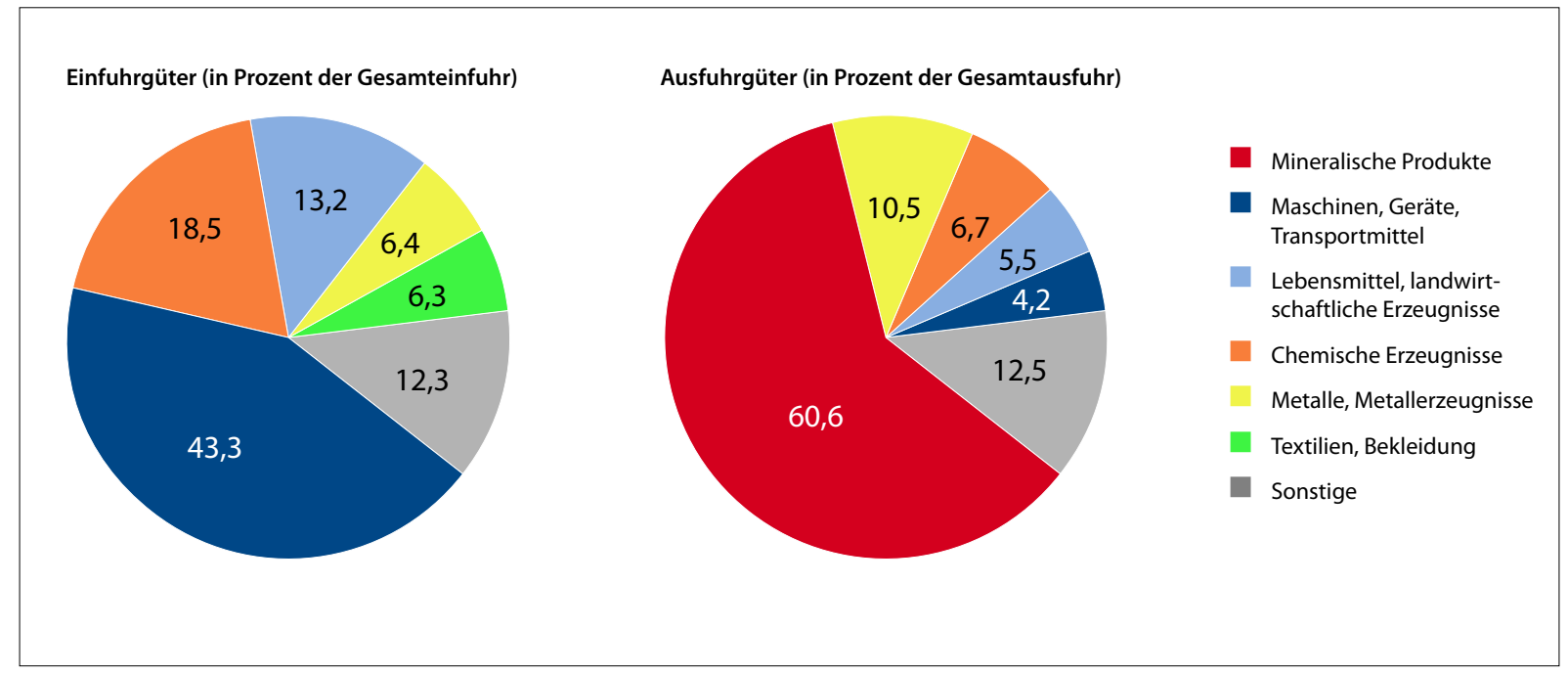

Quelle: German Trade \& Invest, Wirtschaftsdaten Kompakt, Eurasische Wirtschaftsunion (EAWU), 2017, <https://www.gtai.de/ GTAI/Content/DE/Trade/Fachdaten/MKT/2017/02/mkt201702148008_20914_wirtschaftsdaten-kompakt---eurasische-wirtsc haftsunion.pdf?v=6>,S. 4 


\section{Einstellungen zur Eurasischen Wirtschaftsunion}

Grafik 1: Einstellungen zur Mitgliedschaft in der Eurasischen Wirtschaftsunion (EAWU)

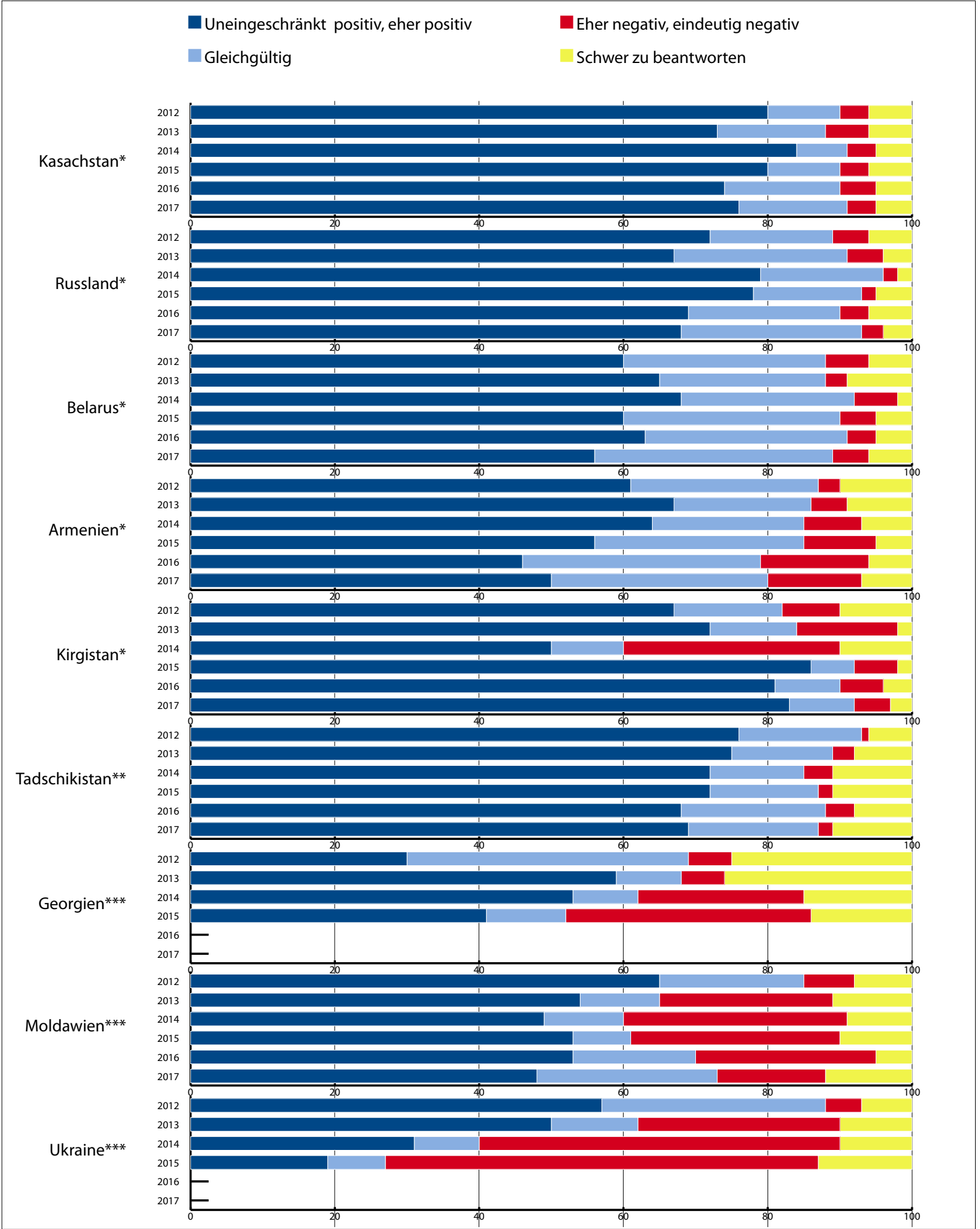

* Mitgliedsstaaten der EAWU; ** EAWU-Beitrittskandidat; *** Staaten, die nicht Mitglied der EAWU sind und ein Assoziierungsabkommen mit der EU unterzeichnet haben

Quelle: Eurasian Development Bank, Integration Barometer 2017, <https://eabr.org/upload/iblock/3ef/EDB-Centre_2017_Report46_EDB-Integration-Barometer_ENG_2.pdf>, S. 9; siehe auch Tabelle 1 auf S. 9/10. 
Tabelle 1: Einstellungen zur Mitgliedschaft in der Eurasischen Wirtschaftsunion (EAWU)

\begin{tabular}{|c|c|c|c|c|c|c|}
\hline & 2012 & 2013 & 2014 & 2015 & 2016 & 2017 \\
\hline \multicolumn{7}{|l|}{ Kasachstan* } \\
\hline $\begin{array}{l}\text { Uneingeschränkt positiv, eher } \\
\text { positiv }\end{array}$ & 80 & 73 & 84 & 80 & 74 & 76 \\
\hline Gleichgültig & 10 & 15 & 7 & 10 & 16 & 15 \\
\hline Eher negativ, eindeutig negativ & 4 & 6 & 4 & 4 & 5 & 4 \\
\hline Schwer zu beantworten & 6 & 6 & 5 & 6 & 5 & 5 \\
\hline \multicolumn{7}{|l|}{ Russland* } \\
\hline $\begin{array}{l}\text { Uneingeschränkt positiv, eher } \\
\text { positiv }\end{array}$ & 72 & 67 & 79 & 78 & 69 & 68 \\
\hline Gleichgültig & 17 & 24 & 17 & 15 & 21 & 25 \\
\hline Eher negativ, eindeutig negativ & 5 & 5 & 2 & 2 & 4 & 3 \\
\hline Schwer zu beantworten & 6 & 4 & 2 & 5 & 6 & 4 \\
\hline \multicolumn{7}{|l|}{ Belarus* } \\
\hline $\begin{array}{l}\text { Uneingeschränkt positiv, eher } \\
\text { positiv }\end{array}$ & 60 & 65 & 68 & 60 & 63 & 56 \\
\hline Gleichgültig & 28 & 23 & 24 & 30 & 28 & 33 \\
\hline Eher negativ, eindeutig negativ & 6 & 3 & 6 & 5 & 4 & 5 \\
\hline Schwer zu beantworten & 6 & 9 & 2 & 5 & 5 & 6 \\
\hline \multicolumn{7}{|l|}{ Armenien* } \\
\hline $\begin{array}{l}\text { Uneingeschränkt positiv, eher } \\
\text { positiv }\end{array}$ & 61 & 67 & 64 & 56 & 46 & 50 \\
\hline Gleichgültig & 26 & 19 & 21 & 29 & 33 & 30 \\
\hline Eher negativ, eindeutig negativ & 3 & 5 & 8 & 10 & 15 & 13 \\
\hline Schwer zu beantworten & 10 & 9 & 7 & 5 & 6 & 7 \\
\hline \multicolumn{7}{|l|}{ Kirgistan* } \\
\hline $\begin{array}{l}\text { Uneingeschränkt positiv, eher } \\
\text { positiv }\end{array}$ & 67 & 72 & 50 & 86 & 81 & 83 \\
\hline Gleichgültig & 15 & 12 & 10 & 6 & 9 & 9 \\
\hline Eher negativ, eindeutig negativ & 8 & 14 & 30 & 6 & 6 & 5 \\
\hline Schwer zu beantworten & 10 & 2 & 10 & 2 & 4 & 3 \\
\hline \multicolumn{7}{|l|}{ Tadschikistan ${ }^{* *}$} \\
\hline $\begin{array}{l}\text { Uneingeschränkt positiv, eher } \\
\text { positiv }\end{array}$ & 76 & 75 & 72 & 72 & 68 & 69 \\
\hline Gleichgültig & 17 & 14 & 13 & 15 & 20 & 18 \\
\hline Eher negativ, eindeutig negativ & 1 & 3 & 4 & 2 & 4 & 2 \\
\hline Schwer zu beantworten & 6 & 8 & 11 & 11 & 8 & 11 \\
\hline
\end{tabular}


Tabelle 1: Einstellungen zur Mitgliedschaft in der Eurasischen Wirtschaftsunion (EAWU) (Fortsetzung)

\begin{tabular}{|c|c|c|c|c|c|c|}
\hline & 2012 & 2013 & 2014 & 2015 & 2016 & 2017 \\
\hline \multicolumn{7}{|l|}{ Georgien*** $^{* *}$} \\
\hline $\begin{array}{l}\text { Uneingeschränkt positiv, eher } \\
\text { positiv }\end{array}$ & 30 & 59 & 53 & 41 & - & - \\
\hline Gleichgültig & 39 & 9 & 9 & 11 & - & \\
\hline Eher negativ, eindeutig negativ & 6 & 6 & 23 & 34 & - & - \\
\hline Schwer zu beantworten & 25 & 26 & 15 & 14 & - & - \\
\hline \multicolumn{7}{|l|}{ Moldawien $^{* * *}$} \\
\hline $\begin{array}{l}\text { Uneingeschränkt positiv, eher } \\
\text { positiv }\end{array}$ & 65 & 54 & 49 & 53 & 53 & 48 \\
\hline Gleichgültig & 20 & 11 & 11 & 8 & 17 & 25 \\
\hline Eher negativ, eindeutig negativ & 7 & 24 & 31 & 29 & 25 & 15 \\
\hline Schwer zu beantworten & 8 & 11 & 9 & 10 & 5 & 12 \\
\hline \multicolumn{7}{|l|}{ Ukraine $^{* * *}$} \\
\hline $\begin{array}{l}\text { Uneingeschränkt positiv, eher } \\
\text { positiv }\end{array}$ & 57 & 50 & 31 & 19 & - & - \\
\hline Gleichgültig & 31 & 12 & 9 & 8 & - & - \\
\hline Eher negativ, eindeutig negativ & 5 & 28 & 50 & 60 & - & - \\
\hline Schwer zu beantworten & 7 & 10 & 10 & 13 & - & - \\
\hline
\end{tabular}

* Mitgliedsstaaten der EAWU; ${ }^{* *}$ EAWU-Beitrittskandidat; ${ }^{* * *}$ Staaten, die nicht Mitglied der EAWU sind und ein Assoziierungsabkommen mit der EU unterzeichnet haben

Quelle: Eurasian Development Bank, Integration Barometer 2017, <https://eabr.org/upload/iblock/3ef/EDB-Centre_2017_Report46_EDB-Integration-Barometer_ENG_2.pdf $>$, 


\section{Das System kollektiver Sicherheit in Eurasien}

Julija Nikitina (Moskauer Staatliche Hochschule für internationale Beziehungen)

\section{Zusammenfassung}

Die Organisation des Vertrags über kollektive Sicherheit (OVKS) gerät oft in Vergessenheit in akademischen und politischen Debatten, obwohl sie die einzige rein militärische Regionalorganisation im postsowjetischen Raum ist, die über ein breites Spektrum an Institutionen zur Reaktion auf unterschiedliche Arten externer Bedrohungen verfügt. Doch es fehlt an einer gemeinsamen Zielsetzung, die die Mitgliedsstaaten mit ihren unterschiedlichen Interessen verbinden könnte. Die Konzentration auf Russland als Zentrum der Integration wird immer unattraktiver. Und auch Russland selbst ist nicht immer bereit, die Rolle des regionalen Hegemons zu übernehmen. Das Hauptzukunftspotenzial der OVKS liegt daher in den Möglichkeiten zur transregionalen Kooperation im Bereich der Bekämpfung neuer Sicherheitsbedrohungen. Ob von diesem Potenzial Gebrauch gemacht wird, ist allerdings alles andere als sicher.

\section{Einleitung}

Wenn europäische Experten sich mit regionalen Organisationen in Eurasien beschäftigen, ist meist von der Eurasischen Wirtschaftsunion (EAWU) die Rede. Zuvor hätte es die Gemeinschaft Unabhängiger Staaten (GUS) sein können - schließlich wurde die Region mitunter auch als "Region der GUS « oder "GUS-Raum» bezeichnet. Das war bis vor Kurzem auch die Bezeichnung der Region in offiziellen Dokumenten der Russischen Föderation. Erst in den letzten Jahren kamen darüber hinaus die Begriffe »eurasische Region" oder "Eurasien" auf (wobei der Begriff „Eurasien« sehr viel früher in Arbeiten westlicher Experten auftauchte, als in russischen Arbeiten oder Beiträgen aus anderen Staaten der Region). Wenn es um China geht, wird oft die Shanghaier Organisation für Zusammenarbeit (SCO) genannt, insbesondere nach dem Beitritt Indiens und Pakistans im Jahr 2017. Dabei gerät unangemessener Weise ein anderer regionaler Zusammenschluss in Vergessenheit: die Organisation des Vertrags über kollektive Sicherheit (OVKS). Sie wird in geopolitischen Diskussionen kaum erwähnt, obwohl sie die einzige rein militärische Organisation im postsowjetischen Raum ist. Warum weckt diese Organisation nicht das Interesse der Politiker und Experten? Dieser Artikel soll zum Verständnis der Evolution dieser Organisation beitragen.

\section{Vom Zusammenbruch der UdSSR bis zur Schaffung eines Systems kollektiver Sicherheit Nach dem Zusammenbruch der Sowjetunion 1991 blieben zwölf ehemalige Unionsrepubliken (ohne die baltischen Staaten, die sich dem Westen zuwandten) mit einer gemeinsamen Infrastruktur sowie mit einem gemeinsamen Devisen- und Wirtschaftsraum, gemein- samen Streitkräften, gemeinsamen Außengrenzen unter gemeinsamem Grenzschutz und als gemeinsamer visa- freier Raum zurück - also in einem Zustand, zu dem}

die Europäische Union erst noch heranzuwachsen hatte. Boris Jelzin erzählte in einem Interview anlässlich des 15. Jahrestages des Zerfalls der UdSSR und der Schaffung der GUS: "Wir versuchten, etwas in der Art der heutigen EU zu schaffen, allerdings mit weniger Bürokratie und Zentralisierung. Wir haben übrigens die ehemaligen Unionsrepubliken all die Jahre finanziell unterstützt, obwohl auch in Russland die wirtschaftliche Lage nicht die beste war. Bundeskanzler Helmut Kohl hat mir seinerzeit gesagt: /Westdeutschland hat Ostdeutschland bis heute noch nicht "verdaut«. Wir aber hatten damals kein ,Westrussland, wir mussten alles selbst machen. Uns war aber bewusst, dass in den Ländern der GUS eine große Zahl unserer Landsleute verblieben war, und die konnten wir nicht ihrem Schicksal überlassen. Deswegen haben wir weiterhin den ehemaligen Unionsrepubliken geholfen«. Entwickelte sich die Europäische Union auf dem Wege der Integration, so gestaltete sich die Entwicklungsbahn der Zusammenarbeit im postsowjetischen Raum komplizierter: Es erfolgte nach einer Integration innerhalb eines Landes (der UdSSR) eine schrittweise Desintegration in den 1990er Jahren und dann wieder eine Reintegration in den 2000er Jahren mit Schaffung der SCO, der OVKS, der Eurasischen Wirtschaftsgemeinschaft und später dann der Eurasischen Wirtschaftsunion (EAWU).

In den 1990er Jahren entwickelte sich die kollektive Sicherheit im organisatorischen Rahmen der GUS: Der Vertrag über kollektive Sicherheit wurde 1992 als eines der Kooperations-Formate der GUS unterzeichnet. Der Beschluss zur Umwandlung dieses Formates in eine vollwertige Organisation erfolgte erst 2002. Gegenwärtig sind Armenien, Belarus, Kasachstan, Kirgistan, Russland und Tadschikistan Mitgliedsstaaten der Organisation des Vertrages über kollektive Sicherheit (OVKS). In den 1990er Jahren bestand das zentrale Kooperationsziel der OVKS in der Verteidigung gegen externe 
Bedrohungen durch Drittstaaten. Darüber hinaus wurden in der "Konzeption für kollektive Sicherheit» von 1995 als Quellen externer Bedrohung neben einem klassischen Angriff von außen auch Versuche der Einmischung in innere Angelegenheiten der Mitgliedsstaaten und der Destabilisierung deren innenpolitischer Lage genannt, ebenso wie internationaler Terrorismus. Diese beiden Bedrohungen standen seinerzeit ganz unten auf der Liste. Gegenwärtig hat sich für die OVKS die Hierarchie der Bedrohungen gewandelt und diese beiden Gefahren gelten oft als wichtigste Bedrohungen für die Sicherheit der OVKS-Mitgliedsstaaten. Hinzugekommen sind der Kampf gegen den Drogenhandel, gegen rechtswidrige Migration aus Drittstaaten sowie Gefahren im Bereich der Informationssicherheit.

Was kann die OVKS in der Praxis übernehmen; über welche Möglichkeiten verfügt sie? Die OVKS kann kollektiv auf externe Sicherheitsbedrohungen reagieren: Das können Angriffe anderer Staaten sein, aber auch solche von terroristischen Organisationen, die in Afghanistan ihre Basis haben und von dort in die Staaten Zentralasiens eindringen. Zur Abwehr eines Angriffs durch Drittstaaten verfügt die OVKS über zwei gemeinsame Verbände der Streitkräfte (Russland-Belarus und Russland-Armenien). In Zentralasien wird diese Funktion von den »Kollektiven Krisenreaktionskräften» (»Kollektiwnyje sily bystrogo raswjortywanija») übernommen (rund 5.000 Mann aus Russland und zentralasiatischen Staaten), die 2001 aufgestellt wurden - vor der Institutionalisierung der OVKS und dem Einsatz der Internationalen Koalition in Afghanistan. Zur Abwendung von Gefahren, die von nichtstaatlichen Akteuren ausgehen von terroristischen und extremistischen Organisationen, von Gruppierungen der organisierten Kriminalität, die von außen angreifen - wurde 2009 die Collective Rapid Reaction Force (russ.: KSOR; dt.: "Kollektive schnelle Einsatzkräfte») aufgestellt, nach unterschiedlichen Einschätzungen zwischen 17.000 und 20.000 Mann aus allen Mitgliedsstaaten, einschließlich eines Polizeikontingents. Zur Regelung zwischenstaatlicher Konflikte wurden die »Kollektive Friedenstruppen« (KMS) der OVKS aufgestellt, die die Zusammenarbeit mit dem UN-Sekretariat in Richtung einer potentiellen Beteiligung an Friedensmissionen unter der Leitung der UNO weiterentwickeln. Sämtliche genannten multinationalen Verbände halten jährlich Manöver ab. Darüber hinaus werden im Rahmen der OVKS Manöver der Sondereinheiten der Innenministerien, sowie Spezialübungen von Einheiten und Mitteln der Militäraufklärung und Manöver von Spezialeinheiten der Drogenpolizeieinheiten der Mitgliedsstaaten abgehalten.

Von den praktischen Aktivitäten der OVKS im Kampf mit den unterschiedlichen Varianten der Bedrohungen für die Sicherheit sind zu nennen:
- die Operation »Kanal« zur Unterbindung des Drogenhandels über die "Nordroute» aus Afghanistan durch Zentralasien und Russland nach Europa;

- die Operation »Illegaler» (russ.: »Nelegal«) zur Bekämpfung rechtswidriger Migration aus Drittstaaten;

- die Operation »PROKSI« (»Protiwodejstwije kriminalu w sfere informacii«; dt.: »Bekämpfung Krimineller im Bereich des Informationswesens«) zur Bekämpfung der Gefahren im IT-Bereich (Sperrung von Internetseiten mit Aufrufen, sich terroristischen Gruppen anzuschließen).

Aus der Beschreibung der Arbeitsbereiche der OVKS ergibt sich, dass die Organisation über ein breites Spektrum an Institutionen zur Reaktion auf unterschiedliche Arten externer Bedrohungen verfügt. Dabei erscheint die OVKS oft, selbst für Russland, von geringerer Bedeutung (im Vergleich zur SCO, zur EAWU und zur BRICS), obwohl diese Organisationen womöglich weniger reale Erfolge vorzuweisen haben. In den Projekten zur Verknüpfung der unterschiedlichen Formate regionaler Zusammenarbeit treten die SCO und die EAWU als Plattformen zur Verbindung mit dem chinesischen Projekt "Neue Seidenstraße« (chin.: "Yídài Yílù») in Erscheinung; die EAWU ist an einer Zusammenarbeit mit der EU interessiert. Die OVKS bleibt dabei außerhalb der russischen Idee einer "Integration der Integrationen" (oder, in anderer Terminologie, des "Großen Eurasischen Projektes«), da diese Ideen vor allem auf eine Entwicklung der Zusammenarbeit in den Bereichen Wirtschaft und Infrastruktur abzielen.

Die OVKS ist daher dazu genötigt, nach anderen Möglichkeiten der internationalen Zusammenarbeit zu suchen. Neben der bereits erwähnten Kooperation mit der UNO arbeitet die OVKS mit der OSZE, der EU, der Organisation für islamische Zusammenarbeit (früher: Organisation der Islamischen Konferenz), der Internationalen Organisation für Migration und dem Internationalen Komitee vom Roten Kreuz zusammen (die Übungen der Friedenstruppen der OVKS finden unter Beteiligung des Internationalen Roten Kreuzes statt). Darüber hinaus hat die OVKS der NATO unterschiedliche Varianten der Zusammenarbeit angeboten, doch ist das nordatlantische Bündnis nicht an einer Zusammenarbeit interessiert und bevorzugt bilaterale Formate mit einzelnen OVKS-Mitgliedsstaaten.

\section{OVKS - ineffektives Projekt einer regionalen Hegemonialmacht?}

Das wenig markante Erscheinungsbild der OVKS und der Unwille der europäischen Staaten, mit der OVKS als Organisation zusammenzuarbeiten, werden durch zwei Arten von Argumenten erklärt: 1) Die OVKS wird von 
Russland dominiert und Moskau unterwirft die Organisation vollständig seinen eigenen Zielsetzungen, daher wäre es einfacher, direkt mit Russland zu verhandeln; 2) die OVKS hat keine realen Einsätze zur Konfliktregulierung durchgeführt, sondern lediglich Übungen abgehalten, und kann daher nicht als effektiv betrachtet werden.

Wenden wir uns dem ersten Argument zu. Bei einer Analyse der Arbeit der regionalen Organisation im postsowjetischen Raum wird Russland praktisch stillschweigend als führende Kraft der Kooperationsprozesse betrachtet, sowohl bei der wirtschaftlichen Integration, als auch im Bereich der Sicherheit. Diese Ansicht wird durch die geopolitische Lage, den Rohstoffreichtum und den Status Russlands als Vetomacht im UN-Sicherheitsrat sowie als Atommacht begründet. Dabei wird die russische Politik im postsowjetischen Raum nicht unbedingt durch diese Faktoren bestimmt; letztere dienen als notwendige Voraussetzung einer Führerschaft in der Region, sind aber ohne den klaren politischen Willen Moskaus, Prozesse der regionalen Zusammenarbeit auf den Weg zu bringen und sich dort tatsächlich einzubringen, nicht wirksam. In der jetzigen Phase ist es für Russland (und womöglich für die übrigen Mitgliedsstaaten auch) interessanter, sich politisch stärker für die Entwicklung von EAWU und SCO zu engagieren, als für die Entwicklung der OVKS.

Eins der wichtigen Probleme für regionale Organisationen im postsowjetischen Raum besteht gegenwärtig in der Suche nach gemeinsamen Zielen. Die Konzentration auf Russland als Zentrum der Integration wird immer unattraktiver. Und auch Russland selbst ist nicht immer bereit, die Rolle des regionalen Hegemons zu übernehmen. Konkrete wirtschaftliche Probleme oder gemeinsame Herausforderungen oder Gefahren funktionieren nicht immer als Anreiz zur Integration. Für eine erfolgreiche regionale Zusammenarbeit sind gemeinsame Werte vonnöten, die alle an der regionalen Integration Beteiligten vereinen würden. Gerade bei der Formulierung von Werten bestehen jedoch Schwierigkeiten. In diesem Zusammenhang wird in den letzten Jahren im Rahmen der OVKS intensiv das Thema Bekämpfung der "farbigen Revolutionen« erörtert. $\mathrm{Zu}$ einem gewissen Grade besteht hierin - neben einer Reihe ernstzunehmender funktionaler Vorteile durch die Zusammenarbeit - für die OVKS-Mitgliedsstaaten die verbindende Idee.

Was das zweite Problem anbelangt, die Effizienz der Organisation, so besteht die wichtigste Frage in der Tat darin, ob die OVKS im Falle einer drastischen Zuspitzung der Lage (und eben nicht nur im Rahmen planmäßiger jährlicher Maßnahmen / Übungen) wirkungsvoll auf regionale Bedrohungen innerer oder äußerer
Natur reagieren kann. Was einen potentiellen Angriff von außen durch terroristische Gruppen aus Afghanistan betrifft, so könnte ein solcher wirksam abgewendet werden: Die OVKS führt seit 2001 das alljährliche Manöver "Rubesh" (dt.: "Grenze») mit einem entsprechenden Szenario durch. Seit 2009 werden nach ähnlichen Szenarien Manöver der Collective Rapid Reaction Force (KSOR) abgehalten. Schwieriger gestaltet sich da die Lage mit den inneren Bedrohungen, mit Versuchen eines Regimewechsels (Regime Change) sowie mit Unruhen und ethnischen Konflikten bzw. Zusammenstößen. In der OVKS ist in den vergangenen Jahren die rechtliche Grundlage wesentlich novelliert worden, auf der nicht nur auf Angriffe von außen, sondern auch auf Krisen und Gefahren für die Stabilität im Innern reagiert werden kann. Die Mechanismen der OVKS können allerdings nur auf Antrag jenes Staates in Gang gesetzt werden, der kollektive Hilfe benötigt. Bislang haben alle Mitgliedsstaaten der OVKS es vorgezogen, mit solchen internen Gefahren für die Stabilität selbständig fertig zu werden.

Konzeptuell ist bei der Einschätzung der Wirksamkeit der kollektiven Sicherheit zu fragen, ob nun die Effektivität zu bewerten ist, mit der eben diese regionale (kollektive) Sicherheit gewährleistet wird, oder aber die Wahrnehmung jedes Mitgliedsstaates hinsichtlich der Sicherheit, die sich aus der Mitgliedschaft in einer regionalen Organisation ergibt. Das kollektive System setzt voraus, dass die Zugehörigkeit zu einer regionalen Organisation jene Probleme löst, die der Mitgliedsstaat nicht allein oder nur mit beträchtlichem Aufwand bewältigen kann.

Auf welche Weise lässt sich nun der Grad des kollektiven Schutzes feststellen? Man könnte meinen, dass die Gewährleistung kollektiver Sicherheit von der Fähigkeit abhängt, kollektiven transnationalen oder grenzüberschreitenden Bedrohungen zu begegnen. Gleichzeitig gibt es eine ganze Reihe Bedrohungen »einzelstaatlicher" Natur: ein Angriff auf einen einzelnen Staat (durch einen anderen Staat oder eine Gruppe von Staaten, wie auch durch eine terroristische Gruppe), oder aber innerstaatliche Krisen (eine politische Destabilisierung, technisch bedingte oder Naturkatastrophen). Bedrohungen auf nationaler Ebene können zweifellos mittelbar auch eine Gefahr für Nachbarstaaten bedeuten (etwa Probleme mit Flüchtlingen), unmittelbar jedoch bedrohen sie nur die Sicherheit des betreffenden Staates. Daher müssen sich die übrigen Mitgliedsstaaten des Systems kollektiver Sicherheit im Klaren sein, weswegen sie sich an diesem System gegenseitiger Hilfe beteiligen. Diese Frage ist eher für die großen Staaten aktuell, die zwar ihren Verbündeten reale Hilfe leisten können, die aber selbst im Falle eines Angriffs kaum auf eine strategisch relevante Hilfe von den anderen Mitgliedsstaaten hoffen können. 
Gibt es nun Probleme der regionalen Sicherheit, die Russland nicht allein oder auf bilateraler Ebene lösen kann? In der Theorie wäre die "gerechteste« Verteilung der Verantwortung in einem System kollektiver Sicherheit dann gegeben, wenn die Ressourcen und Möglichkeiten der Mitgliedsstaaten ungefähr gleich sind. In der OVKS besteht ein eindeutiges »Übergewicht« Russlands. Daher sollte Moskau sich eindeutig bewusst sein, warum Russland sich an einem System kollektiver Sicherheit beteiligt. Europäische Experten äußern in informellen Gesprächen oft ihr Unverständnis darüber, wozu denn eigentlich Russland bestimmte kollektive Strukturen im postsowjetischen Raum brauche, da es ja von den Ländern des Westens bereits als "vollwertiger" regionaler Akteur im Sicherheitsbereich wahrgenommen wird.

\section{Möglichkeiten für interregionale Zusammenarbeit}

Ungeachtet des Unwillens zur Zusammenarbeit und der Zweifel an der Effektivität der OVKS gibt es in der gegenwärtigen Phase einen Bereich, der nicht nur für die OVKS-Staaten, sondern auch für die Länder der EU und der NATO von Interesse ist. Das ist der Kampf gegen Extremismus und Terrorismus, und das Problem der Propaganda durch extremistische Gruppen in den sozialen Medien. Hinzu kommt das Problem der Rückkehrer, die außerhalb ihrer Herkunftsländer an bewaffneten Konflikten beteiligt waren. In diesem Bereich bestehen viele Möglichkeiten zum Erfahrungsaustausch, vor allem über die Linie der OSZE.

Die OVKS könnte beispielsweise ihre Erfahrungen aus der Operation PROKSI teilen, bei der es um die Identifizierung und Sperrung von Internetseiten mit extremistischem Inhalt ging, die unter anderem zur Rekrutierung genutzt werden. Die Operation wurde 2009 durchgeführt, es wurden erhebliche Erfahrungen gewonnen, die auch für andere Länder von Nutzen sein können. Natürlich ließe sich dieser Ansatz bis zu einem gewissen Grad als reaktiv bezeichnen, weil in die- sem Rahmen nicht die Gründe für Extremismus in der Region bekämpft werden. Sicherheitsorganisationen sollen allerdings in der Regel nicht die Ursachen bekämpfen, die den verschiedenen Bedrohungen zugrunde liegen. Vielmehr ist eine ihrer zentralen Funktionen, die Bedrohungen zurückzudrängen und ihnen vorzubeugen. Was die OSZE anbelangt, so gibt es in deren Rahmen die Praxis, sogenannte Manuals zur Bekämpfung von Extremismus und Terrorismus zusammenzustellen, etwa zur Bekämpfung auf der Ebene lokaler Gemeinschaften oder durch eine Zusammenarbeit der Polizei mit diesen Gemeinschaften, um eine Verbreitung von Extremismus und Terrorismus zu verhindern. Die OVKS könnte also ihrerseits diese Erfahrung übernehmen und diese Manuals für die Bedürfnisse ihrer Mitgliedsstaaten adaptieren (alle OVKS Staaten sind Mitglieder der OSZE).

Auch wenn die OVKS, wie bereits erwähnt, aus Projekten der regionsübergreifenden Zusammenarbeit im Rahmen der Idee von einer "Integration der Integrationen « im wirtschaftlichen Bereich gewissermaßen herausfällt und für Russland im Vergleich zu EAWU und SCO ein weniger vordringliches regionales Projekt darstellt, bedeutet das nicht, dass die OVKS irgendeine marginale Organisation ohne internationales Kooperationspotenzial wäre: Die Liste der internationalen Kontakte der OVKS ist recht lang. Wie oben bereits erwähnt, gibt es eine aktive Zusammenarbeit mit der OSZE, was die Verbreitung einheitlicher Ansätze zur Bekämpfung der verschiedenen Herausforderungen und Bedrohungen auf dem gesamten OSZE-Gebiet befördert. Angesichts der Krise in den Beziehungen zwischen Russland und dem Westen ist es nun wichtig, Dialogplattformen zu den wichtigsten Sicherheitsfragen aufrechtzuerhalten, damit einheitliche, auf dem gesamten Kontinent gültige Standards zur Bewältigung der neuen Herausforderungen und Bedrohungen verfolgt werden.

Übersetzung aus dem Russischen: Hartmut Schröder

\section{Über die Autorin}

Julija Nikitina ist Dozentin am Lehrstuhl für weltpolitische Prozesse an der Moskauer Staatlichen Hochschule für internationale Beziehungen (MGIMO)

\section{Lesetipps}

- Baev, Pavel: The CSTO: Military Dimensions of the Russian Reintegration Effort, in: S. F. Starr, S. V. Cornell (Hg.): Putin's Grand Strategy: The Eurasian Union and Its Discontents, Washington D.C. \& Stockholm-Nacka, 2014, S. 40-48; <https://www.silkroadstudies.org/resources/pdf/publications/4-1409GrandStrategy-Baev.pdf>.

- Ban Ki-moon. Remarks to Collective Security Treaty Organization [as delivered], 22. April 2011; <https://www. un.org/sg/en/content/sg/speeches/2011-04-22/remarks-collective-security-treaty-organization-delivered>.

- Bordyuzha, Nikolai: The Collective Security treaty Organization: A Brief Overview, in: Ursel Schlichting (Hg.): OSCE Yearbook 2010 (S. 339-350); <https://ifsh.de/file-CORE/documents/yearbook/english/10/Bordyuzha-en. $\mathrm{pdf}>$. 
- Nikitina, Yulia: The Collective Security Treaty Organization Through the Looking Glass, in: Problems of PostCommunism, 59.2014, Nr. 3, S. 41-52; <https://www.tandfonline.com/doi/abs/10.2753/PPC1075-8216590304>.

- Omelicheva, Mariya: Eurasia's CSTO and SCO: A Failure to Address the Trafficking/Terrorism Nexus [PONARS Eurasia policy memo Nr. 455], Januar 2017; <http://www.ponarseurasia.org/memo/ eurasia-csto-and-sco-failure-address-trafficking-terrorism-nexus $>$.

\section{STATISTIK}

\section{Militärausgaben der OVKS-Mitgliedsstaaten}

Tabelle 1: Militärausgaben der OVKS-Staaten (Mio. US-Dollar, konstant 2015)

\begin{tabular}{|l|r|r|r|r|r|r|r|}
\hline & \multicolumn{1}{|c|}{2010} & \multicolumn{1}{c|}{2011} & \multicolumn{1}{c|}{2012} & 2013 & \multicolumn{1}{c}{2014} & \multicolumn{1}{c}{2015} & 2016 \\
Armenien & 385 & 353 & 362 & 407 & 413 & 447 & 423 \\
Kasachstan & 1.369 & 1.510 & 1.764 & 1.992 & 1.988 & 2.046 & 1.504 \\
Kirgistan & 192 & 189 & 189 & 202 & 233 & $231^{*}$ & $212^{*}$ \\
Russland & $44.338^{*}$ & $47.321^{*}$ & $54.832^{*}$ & 57.500 & 61.622 & 66.419 & 70.345 \\
Tadschikistan & 53,5 & 66,5 & 69,1 & - & 88 & 95,8 & - \\
Belarus & 556 & 597 & 678 & 726 & 737 & 724 & 664 \\
NATO insgesamt** & 1.013 .705 & 1.044 .467 & 996.595 & 968.518 & 942.294 & 895.059 & 911.658 \\
\hline
\end{tabular}

* SIPRI-Schätzungen; ${ }^{* *}$ NATO-Militärausgaben: Mio. US-Dollar, konstant 2010

Quelle: SIPRI Military Expenditure Database 2017, <https://www.sipri.org/sites/default/files/SIPRI-Milex-data-1949-2016.xlsx>; NATO Defence Expendtiture of NATO Countries (2010-2017) 2018, <https://www.nato.int/docu/pr/2018/pr-cp2018-16.xlsx>

\section{Grafik 1: Militärausgaben der OVKS-Staaten (2002-2016, in Prozent des BIP)}

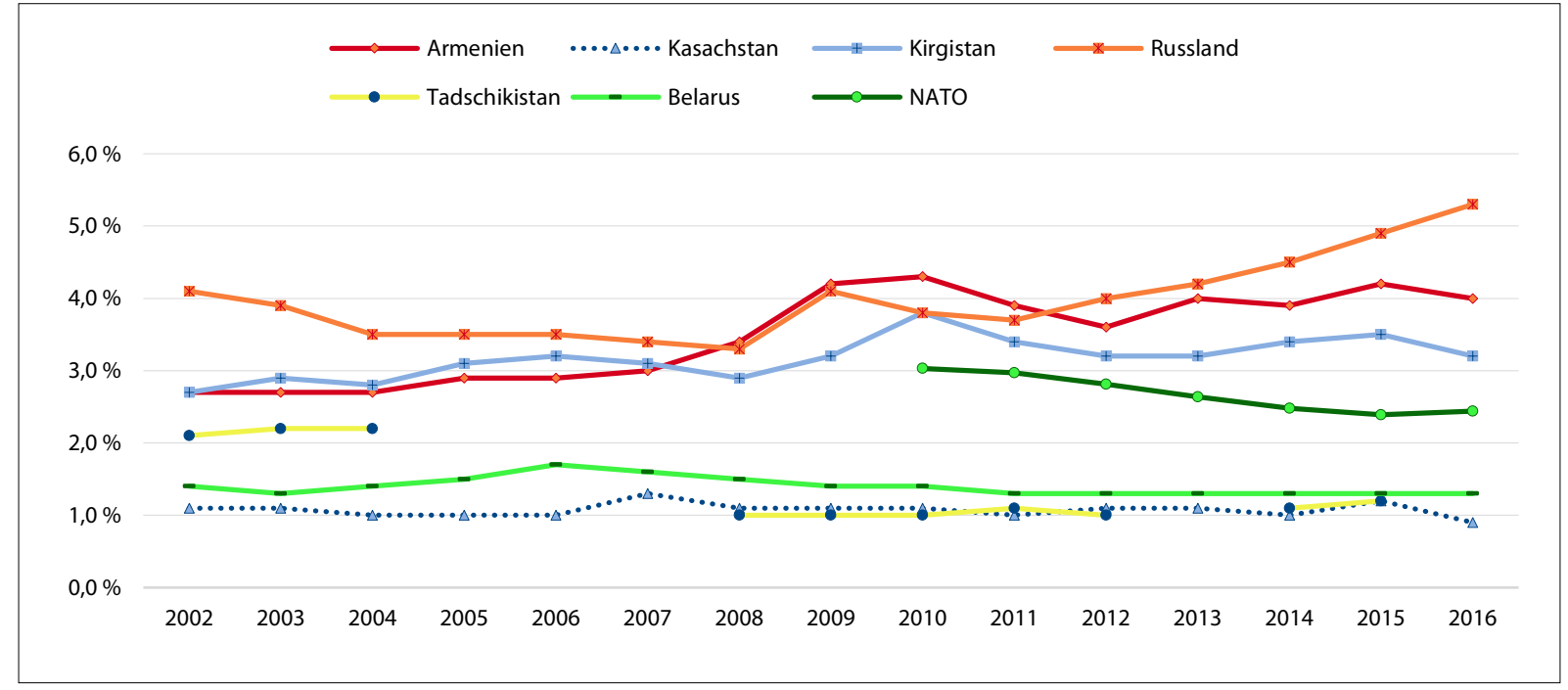

Quelle: SIPRI Military Expenditure Database 2017, <https://www.sipri.org/sites/default/files/SIPRI-Milex-data-1949-2016.xlsx>; NATO Defence Expendtiture of NATO Countries (2010-2017) 2018, <https://www.nato.int/docu/pr/2018/pr-cp2018-16.xlsx> 
Tabelle 2: Militärausgaben der OVKS-Staaten (2002-2016, in Prozent des BIP)

\begin{tabular}{|c|c|c|c|c|c|c|c|c|c|c|c|c|c|c|c|}
\hline & 2002 & 2003 & 2004 & 2005 & 2006 & 2007 & 2008 & 2009 & 2010 & 2011 & 2012 & 2013 & 2014 & 2015 & 2016 \\
\hline Armenien & $2,7 \%$ & $2,7 \%$ & $2,7 \%$ & $2,9 \%$ & $2,9 \%$ & $3,0 \%$ & $3,4 \%$ & $4,2 \%$ & $4,3 \%$ & $3,9 \%$ & $3,6 \%$ & $4,0 \%$ & $3,9 \%$ & $4,2 \%$ & $4,0 \%$ \\
\hline Kasachstan & $1,1 \%$ & $1,1 \%$ & $1,0 \%$ & $1,0 \%$ & $1,0 \%$ & $1,3 \%$ & $1,1 \%$ & $1,1 \%$ & $1,1 \%$ & $1,0 \%$ & $1,1 \%$ & $1,1 \%$ & $1,0 \%$ & $1,2 \%$ & $0,9 \%$ \\
\hline Kirgistan & $2,7 \%$ & $2,9 \%$ & $2,8 \%$ & $3,1 \%$ & $3,2 \%$ & $3,1 \%$ & $2,9 \%$ & $3,2 \%$ & $3,8 \%$ & $3,4 \%$ & $3,2 \%$ & $3,2 \%$ & $3,4 \%$ & $3,5 \%$ & $3,2 \%$ \\
\hline Russland & $4,1 \%$ & $3,9 \%$ & $3,5 \%$ & $3,5 \%$ & $3,5 \%$ & $3,4 \%$ & $3,3 \%$ & $4,1 \%$ & $3,8 \%$ & $3,7 \%$ & $4,0 \%$ & $4,2 \%$ & $4,5 \%$ & $4,9 \%$ & $5,3 \%$ \\
\hline ikistan & $2,1 \%$ & $2,2 \%$ & $2,2 \%$ & - & - & - & $1,0 \%$ & $1,0 \%$ & $1,0 \%$ & $1,1 \%$ & $1,0 \%$ & - & $1,1 \%$ & $1,2 \%$ & - \\
\hline Belarus & $1,4 \%$ & $1,3 \%$ & $1,4 \%$ & $1,5 \%$ & $1,7 \%$ & $1,6 \%$ & $1,5 \%$ & $1,4 \%$ & $1,4 \%$ & $1,3 \%$ & $1,3 \%$ & $1,3 \%$ & $1,3 \%$ & $1,3 \%$ & $1,3 \%$ \\
\hline NATO & & & & & & & & & $3,0 \%$ & $3,0 \%$ & $2,8 \%$ & $2,6 \%$ & $2,5 \%$ & $2,4 \%$ & $2,4 \%$ \\
\hline
\end{tabular}

Quelle: SIPRI Military Expenditure Database 2017, <https://www.sipri.org/sites/default/files/SIPRI-Milex-data-1949-2016.xlsx>; NATO Defence Expendtiture of NATO Countries (2010-2017) 2018, <https://www.nato.int/docu/pr/2018/pr-cp2018-16.xlsx>

UMFRAGE

\section{Einstellung der russischen Bevölkerung zum Fall Skripal}

Grafik 1: Haben Sie von der Vergiftung des ehemaligen Geheimdienstagenten Sergej Skripal und seiner Tochter gehört?

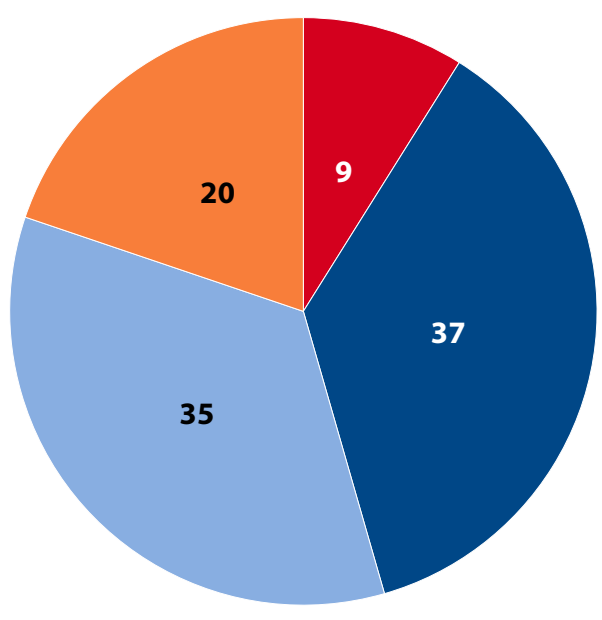

- Ich verfolge die Situation mit großer Aufmerksamkeit

- Ich habe viel davon gehört

Ich habe schon was davon gehört, weiß aber nicht genau, worum es geht

- Ich höre zum ersten Mal davon

Anmerkung: Durch einen Rundungsfehler weicht die Gesamtanzahl der Respondenten von $100 \%$ ab.

Quelle: repräsentative Umfrage des Levada-Zentrums vom 23-27 März, 2018, <https://www.levada.ru/2018/04/05/delo-skripa lyal>, veröffentlicht am 5. April 2018 
Grafik 2: Einige westliche Staaten beschuldigen Russland des Mordsversuchs an Sergej Skripal. Denken Sie, dass diese Beschuldigungen begründet sind?

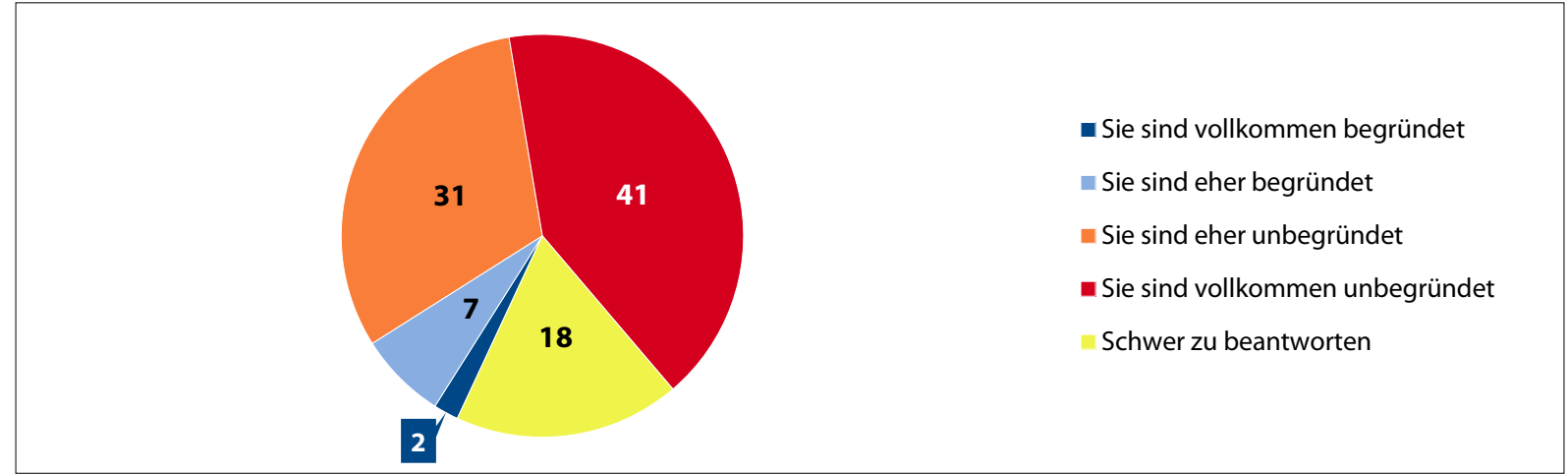

Anmerkung: Durch einen Rundungsfehler weicht die Gesamtanzahl der Respondenten von $100 \%$ ab.

Quelle: repräsentative Umfrage des Levada-Zentrums vom 23-27 März, 2018, <https://www.levada.ru/2018/04/05/delo-skripa lyal>, veröffentlicht am 5. April 2018

Grafik 3: Was war Ihrer Meinung nach der Hauptgrund für die Ausweisung russischer Diplomaten?

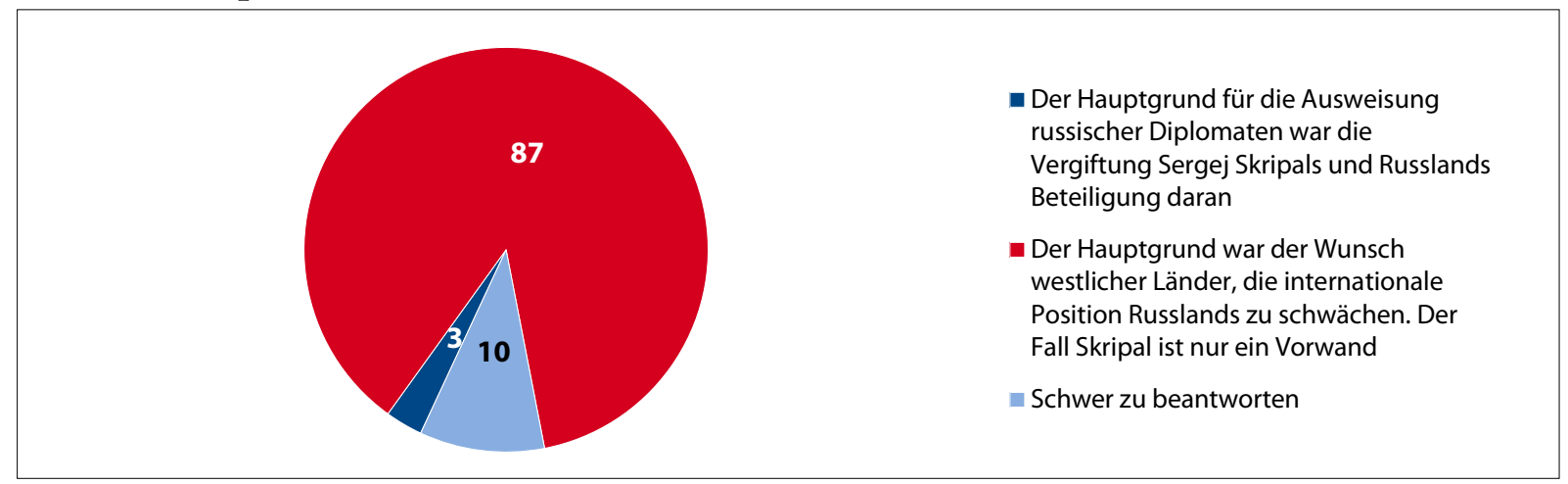

Quelle: WZIOM-Umfrage, <https://wciom.ru/index.php?id=236\&uid=9044>, veröffentlicht am 13. April 2018

Grafik 4: Denken Sie, dass der Konflikt um den Fall Skripal sich in den nächsten sechs Monaten verschärfen oder eher abklingen wird?

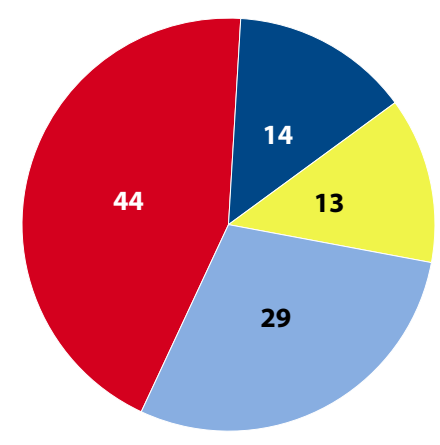

- Der Konflikt wird abklingen

- Der Konflikt wird sich verschärfen

Schwer zu beantworten

Keine Kenntnis vom Fall Skripal

Quelle: FOM-Umfrage, <http://fom.ru/Bezopasnost-i-pravo/14003>, veröffentlicht am 10. April 2018 


\section{3. - 14. April 2018}

\begin{tabular}{|c|c|}
\hline 03.04 .2018 & $\begin{array}{l}\text { Mitglieder des Rates für die Entwicklung der Zivilgesellschaft und für Menschenrechte beim Prä- } \\
\text { sidenten der Russischen Föderation wenden sich an die Medienaufsichtsbehörde »Roskomnadsor» } \\
\text { mit der Bitte, den Messenger-Dienst »Telegram« nicht zu blockieren. »Telegram« war am 20. März } \\
\text { vom Obersten Gerichtshof der Russischen Föderation dazu verurteilt worden, binnen } 15 \text { Tagen } \\
\text { seine Dechiffrierschlüssel an den Inlandsgeheimdienst FSB zu übergeben. Die Frist zur Heraus- } \\
\text { gabe läuft am 4. April ab. }\end{array}$ \\
\hline 04.04 .2018 & $\begin{array}{l}\text { Im Rahmen seines zweitägigen Besuches in Ankara verkündet der russische Präsident Wladimir } \\
\text { Putin, die vereinbarte Lieferung von Flugabwehr-Raketensystemen zu beschleunigen. Neuer Liefer- } \\
\text { zeitpunkt sei nun Juli 2019. Darüber hinaus geben die beiden Präsidenten Putin und Erdoğan auf } \\
\text { einer feierlichen Zeremonie das Zeichen zum Baubeginn des mit russischer Hilfe geplanten türki- } \\
\text { schen Atomkraftwerks Akkuyu, das ab } 2023 \text { in Betrieb gehen soll. }\end{array}$ \\
\hline 04.04 .2018 & $\begin{array}{l}\text { Im Rahmen der Ermittlungen zum Brand eines Einkaufszentrums in Kemerowo am 25. März wird } \\
\text { das Wohnhaus des Leiters des Katastrophenschutzministeriums der Region, Alexander Mamon- } \\
\text { tow, durchsucht. }\end{array}$ \\
\hline 04.04 .2018 & $\begin{array}{l}\text { In Ankara findet unter Beteiligung des russischen Präsidenten Putin, des türkischen Präsidenten } \\
\text { Erdoğan und des iranischen Präsidenten Rouhani ein Gipfeltreffen zur Lage in Syrien statt, dabei wird } \\
\text { zu einer Verbesserung der humanitären Lage dort und zu einer anhaltenden Waffenruhe aufgerufen. }\end{array}$ \\
\hline 04.04 .2018 & $\begin{array}{l}\text { Alexander Kogan, Minister für Ökologie und Naturschutz in der Region Moskau, gibt bekannt, } \\
\text { dass auf dem Gelände der Deponie "Jadrowo« in Wolokolamsk eine moderne Müllbeseitigungs- } \\
\text { anlage gebaut werden wird. Weitere fünf Deponien in der Region werden modernisiert und mit } \\
\text { modernen Abluftanlagen ausgestattet. Im März kam es immer wieder zu erhöhten Konzentratio- } \\
\text { nen von Schwefelwasserstoff in der Luft. Die Einwohner von Wolokolamsk hatten daraufhin mehr- } \\
\text { mals Proteste organisiert. }\end{array}$ \\
\hline 05.04 .2018 & $\begin{array}{l}\text { Der US-Fernsehsender CNN berichtet, dass drei russische Oligarchen während ihres USA-Besu- } \\
\text { ches kontrolliert worden seien. Die Kontrollen gehen auf die Ermittlungen des US-Sonderermitt- } \\
\text { lers Robert Mueller zurück. Dieser ermittelt wegen des Verdachts auf eine Einmischung Russlands } \\
\text { in die US-Präsidentschaftswahlen im Jahr } 2016 \text {. }\end{array}$ \\
\hline 05.04 .2018 & $\begin{array}{l}\text { Der Leiter der Menschenrechtsorganisation "Memorial« in Karelien, Jurij Dmitrijew, wird vom Vor- } \\
\text { wurf der Kinderpornographie freigesprochen. Das städtische Gericht in Petrosawodsk entschied } \\
\text { gegen den Antrag der Staatsanwaltschaft auf neun Jahre Lagerhaft, sondern verurteilte Dmitri- } \\
\text { jew lediglich zu einer Haftstrafe von drei Monaten wegen des illegalen Besitzes einer Schrotflinte. } \\
\text { Dmitrijew war im Dezember } 2016 \text { verhaftet und im Januar } 2018 \text { aus der Untersuchungshaft ent- } \\
\text { lassen worden. }\end{array}$ \\
\hline 06.04 .2018 & $\begin{array}{l}\text { Die Medienaufsichtsbehörde »Roskomnadsor« reicht beim Taganskij-Bezirksgericht Klage auf Sper- } \\
\text { rung des Messenger-Dienstes »Telegram« ein. »Telegram« hatte die 15-tägige Frist zur Herausgabe } \\
\text { seines Dechiffrierschlüssels an den FSB am } 4 \text {. April verstreichen lassen. Damit verstößt der Dienst } \\
\text { nach Ansicht von »Roskomnadsor« gegen das »Gesetz über die Bereitstellung von Dechiffrierschlüs- } \\
\text { seln zur Entschlüsselung von Nachrichten«. }\end{array}$ \\
\hline 06.04 .2018 & $\begin{array}{l}\text { Die USA verhängen weitere Sanktionen gegen russische Geschäftsleute und Regierungsvertreter. } \\
\text { Auf der von der US-Regierung veröffentlichten Liste stehen } 17 \text { ranghohe Vertreter der russischen } \\
\text { Regierung, sieben Oligarchen (darunter unter anderem Oleg Deripaska und Viktor Wekselberg) } \\
\text { und } 14 \text { Unternehmen. Mit dieser Maßnahme werden alle Vermögenswerte der Betroffenen in den } \\
\text { USA eingefroren. Außerdem ist es US-Bürgern untersagt, mit ihnen Geschäfte zu tätigen. }\end{array}$ \\
\hline 08.04 .2018 & $\begin{array}{l}\text { Die USA machen Russland für einen möglichen Chemiewaffenangriff in der syrischen Stadt Duma } \\
\text { vom 6. April verantwortlich. Jurij Jewtuschenko, Leiter des russischen "Zentrums zur Aussöhnung } \\
\text { der Konfliktparteien in Syrien«, widersprach der Darstellung, es habe sich um einen Angriff mit } \\
\text { chemischen Waffen gehandelt. }\end{array}$ \\
\hline
\end{tabular}




\begin{tabular}{|c|c|}
\hline 09.04 .2018 & $\begin{array}{l}\text { Der russische Aktienindex RTS sinkt um zwölf Prozent. Dies ist der größte Tagesverlust seit etwa } \\
\text { vier Jahren. Größter Verlierer ist der Aluminiumhersteller »Rusal«, dessen Aktien fast ein Viertel } \\
\text { ihres Wertes verloren. Zuvor hatte der Konzern mitgeteilt, dass die neuen US-Sanktionen sich nega- } \\
\text { tiv auf das Geschäft auswirken könnten. Der Oligarch Oleg Deripaska hält den größten Anteil der } \\
\text { Aktien des Konzerns und steht auf der Sanktionsliste der USA. }\end{array}$ \\
\hline 09.04 .2018 & $\begin{array}{l}\text { Der Pressesprecher des russischen Präsidenten, Dmitrij Peskow, bezeichnet die von den USA vorge- } \\
\text { brachte Beschuldigung, die regierungsnahe Kriegspartei in Syrien hätte bei ihrem Angriff in Duma } \\
\text { Chemiewaffen eingesetzt, als »Provokation«. }\end{array}$ \\
\hline 09.04 .2018 & $\begin{array}{l}\text { In einem Telefonat bekräftigen die deutsche Bundeskanzlerin Angela Merkel und der russische } \\
\text { Präsident Wladimir Putin den Wunsch einer weiteren engen Zusammenarbeit im Hinblick auf die } \\
\text { Krise um die Ukraine. Beide Seiten betonen die Bedeutung des sogenannten „Normandie-Formats" } \\
\text { sowie eine Neuauflage des Minsker Abkommens zur Lösung des Konflikts. }\end{array}$ \\
\hline 10.04 .2018 & $\begin{array}{l}\text { Julija Skripal, Tochter des Ex-Doppelagenten Sergej Skripal, die gemeinsam mit ihrem Vater Anfang } \\
\text { März Opfer eines Giftgasanschlags geworden war, wird aus dem Krankenhaus entlassen. }\end{array}$ \\
\hline 11.04 .2018 & $\begin{array}{l}\text { US-Präsident Donald Trump kündigt via Twitter als Vergeltung für den mutmaßlichen Giftgas- } \\
\text { einsatz im syrischen Duma einen Raketenangriff an. Marija Sacharowa, Sprecherin des russischen } \\
\text { Außenministeriums, vermutet daraufhin, die USA wollten Beweise im Zusammenhang mit dem } \\
\text { Angriff zerstören. Donald Trump solle die Raketen lieber zur Bekämpfung des Terrorismus nut- } \\
\text { zen, als sie auf die »legitime Regierung Syriens« zu richten, die versuche, den Terrorismus im Land } \\
\text { zu bekämpfen, so Sacharowa. }\end{array}$ \\
\hline 12.04 .2018 & $\begin{array}{l}\text { Das Stadtgericht Moskau bestätigt das im Dezember gegen Alexej Uljukajew ergangene Urteil. } \\
\text { Uljukajew, ehemaliger Minister für wirtschaftliche Entwicklung, war wegen Korruption zu acht } \\
\text { Jahren Haft und einer Geldstrafe von } 130 \text { Millionen Rubel (etwa 1,7 Mio. Euro) verurteilt. Er soll } \\
\text { den Ölkonzern »Rosneft« um zwei Millionen US-Dollar erpresst haben, als dieser im Zuge der Pri- } \\
\text { vatisierung Anteile des Staatskonzerns »Baschneft« erwerben wollte. Igor Setschin, Chef von »Ros- } \\
\text { neft«, sagt nun aus, dass Uljukajew eigentlich gar nicht die Macht gehabt habe, die Privatisierung } \\
\text { von "Baschneft« zu beeinflussen. Er habe von ihm, Setschin, im Laufe eines etwa zehn Minuten } \\
\text { währenden Gesprächs jedoch trotzdem ein Bestechungsgeld erpresst. Setschin war bereits zuvor als } \\
\text { Zeuge geladen gewesen, bislang jedoch nie vor Gericht erschienen. }\end{array}$ \\
\hline 12.04 .2018 & $\begin{array}{l}\text { Die »Organisation für das Verbot chemischer Waffen« (OPCW) bestätigt in ihrem Untersuchungs- } \\
\text { bericht, dass für den Giftgasanschlag auf den Ex-Doppelagenten Sergej Skripal und dessen Toch- } \\
\text { ter Julija im März das Nervengift »Nowitschok» verwendet worden sei. Laut Bericht der OPCW } \\
\text { sei das Gift von hoher Reinheit gewesen, es wurden keine Verunreinigungen nachgewiesen. Zeit- } \\
\text { gleich berichtet der britische Fernsehsender "Sky News« über Erkenntnisse der britischen Ermitt- } \\
\text { lungsbehörden darüber, dass Russland Skripal seit } 2013 \text { habe beschatten lassen. }\end{array}$ \\
\hline 14.04 .2018 & $\begin{array}{l}\text { Die USA, Frankreich und Großbritannien greifen als Reaktion auf den mutmaßlichen Giftgasan- } \\
\text { griff auf die Stadt Duma am 6. April gezielt Ziele in Syrien an. Die Raketen treffen ein Forschungs- } \\
\text { zentrum in Damaskus und ein Chemiewaffenlager in Homs. Konstantin Kossatschew, Vorsitzen- } \\
\text { der des Ausschusses für internationale Angelegenheit beim Föderationsrat nennt den Angriff eine } \\
\text { Verletzung internationalen Rechts und unbegründeten Angriff auf einen souveränen Staat. }\end{array}$ \\
\hline 14.04 .2018 & $\begin{array}{l}\text { Erneut finden in Wolokolamsk, unweit der Deponie "Jadrowo«, aus der gesundheitsschädliche } \\
\text { Gase ausgetreten waren, Protestkundgebungen statt. Nach Angaben der Organisatoren nehmen } \\
\text { an den Protesten mehr als } 500 \text { Menschen teil. Die Menschen fordern die vollständige Schließung } \\
\text { der Deponie, nicht nur, wie geplant, ihre Modernisierung. Auch in mehreren anderen Städten der } \\
\text { Region Moskau finden Demonstrationen für die Schließung verschiedener Müll-Deponien statt. }\end{array}$ \\
\hline
\end{tabular}




\begin{tabular}{|l|l|}
\hline 14.04.2018 & $\begin{array}{l}\text { Der russische Außenminister Sergej Lawrow wirft der »Organisation für das Verbot chemischer } \\
\text { Waffen (OPCW) vor, ihren Bericht über das bei dem Nervengasanschlag auf Ex-Doppelagent Ser- } \\
\text { gej Skripal und seine Tochter Julija im März im britischen Salisbury verwendete Gift, manipuliert } \\
\text { zu haben. Laut Lawrow habe das Schweizer Labor, das die Blutproben der beiden Opfer untersucht } \\
\text { habe, Spuren des chemischen Kampfstoffes BZ nachgewiesen, der zu keiner Zeit von der Sowjet- } \\
\text { union oder Russland, wohl aber vom Westen eingesetzt worden sei. }\end{array}$ \\
\hline
\end{tabular}

Die Chronik wird zeitnah erstellt und basiert ausschließlich auf im Internet frei zugänglichen Quellen. Die Redaktion der RusslandAnalysen kann keine Gewähr für die Richtigkeit der Angaben übernehmen.

Zusammengestellt von Alena Göbel

Sie können die gesamte Chronik seit 1964 auch auf <http://www.laender-analysen.de/russland/> unter dem Link»Chronik«lesen.

Herausgeber:

Forschungsstelle Osteuropa an der Universität Bremen

Deutsche Gesellschaft für Osteuropakunde e.V.

Deutsches Polen-Institut

Leibniz-Institut für Agrarentwicklung in Transformationsökonomien

Leibniz-Institut für Ost- und Südosteuropaforschung

Zentrum für Osteuropa- und internationale Studien (ZOiS) gGmbH

Redaktion:

Evgeniya Bakalova (verantwortlich) und Anastasia Stoll

Sprachredaktion: Hartmut Schröder

Chronik: Alena Göbel

Satz: Matthias Neumann

Wissenschaftlicher Beirat:

Dr. Sabine Fischer, Stiftung Wissenschaft und Politik, Berlin

Prof. Dr. Alexander Libman, Universität München

Prof. Dr. Jeronim Perović, Universität Zürich

Dr. Cindy Wittke, Leibniz-Institut für Ost- und Südosteuropaforschung Regensburg

Die Meinungen, die in den Russland-Analysen geäußert werden, geben ausschließlich die Auffassung der Autoren wieder.

Abdruck und sonstige publizistische Nutzung sind nach Rücksprache mit der Redaktion gestattet.

Russland-Analysen-Layout: Cengiz Kibaroglu, Matthias Neumann und Michael Clemens

Alle Ausgaben der Russland-Analysen sind mit Themen- und Autorenindex archiviert unter www.laender-analysen.de

Die Russland-Analysen werden im Rahmen eines Lizenzvertrages in das Internetangebot der Bundeszentrale für politische Bildung (www.bpb.de) aufgenommen.

ISSN 1613-3390 @ 2018 by Forschungsstelle Osteuropa an der Universität Bremen

Forschungsstelle Osteuropa • Länder-Analysen • Klagenfurter Str. 8 • 28359 Bremen • Telefon: + 49 421-218-69600 • Telefax: +49 421-218-69607

e-mail: laender-analysen@uni-bremen.de•Internet-Adresse: http://www.laender-analysen.de/russland/ 


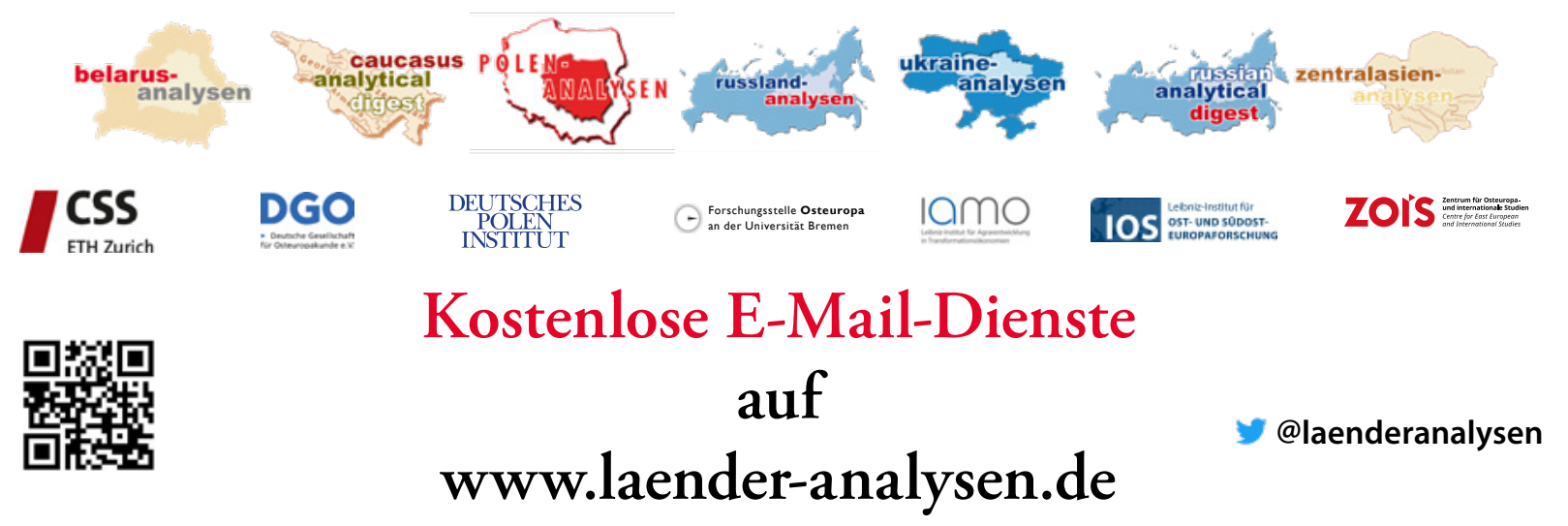

Die Länder-Analysen bieten regelmäßig im kostenlosen Abonnement kompetente Einschätzungen aktueller politischer, wirtschaftlicher, sozialer und kultureller Entwicklungen in Ostmitteleuropa und der GUS. Alle Länder-Analysen verstehen sich als Teil eines gemeinsamen Projektes, das der wissenschaftlich fundierten, allgemeinverständlich formulierten Analyse der Entwicklungen im östlichen Europa, der Offenheit für verschiedene inhaltliche Positionen und der kostenlosen und nicht-kommerziellen Information einer breit verstandenen interessierten Öffentlichkeit verpflichtet ist. Autor/innen sind internationale Fachwissenschaftler/innen und Expert/innen. Die Redaktionen der Länder-Analysen bestehen aus Wissenschaftler/innen mit langjähriger Forschungserfahrung.

Die deutschsprachigen Länder-Analysen werden gemeinsam von der Forschungsstelle Osteuropa an der Universität Bremen, dem Zentrum für Osteuropa- und internationale Studien, der Deutschen Gesellschaft für Osteuropakunde, dem Deutschen Polen-Institut, dem Leibniz-Institut für Agrarentwicklung in Transformationsökonomien und dem Leibniz-Institut für Ost- und Südosteuropaforschung herausgegeben. Die englischsprachigen Länder-Analysen erscheinen in Kooperation der Forschungsstelle Osteuropa mit dem Center for Security Studies (CSS) der ETH Zürich.

Die Länder-Analysen bieten regelmäßig Kurzanalysen zu aktuellen Themen, ergänzt um Grafiken und Tabellen sowie Dokumentationen. Zusätzlich gibt es eine Chronik aktueller Ereignisse. Alle Länder-Analysen sind auch mit Archiv und Indizes online verfügbar unter <www.laender-analysen.de $>$.

\section{Belarus-Analysen}

Erscheinungsweise: zweimonatlich

Abonnement unter: <http://www.laender-analysen.de/belarus/>

\section{Caucasus Analytical Digest}

In englischer Sprache. Erscheinungsweise: monatlich

Abonnement unter: <http://www.css.ethz.ch/en/publications/cad.html>

\section{Polen-Analysen}

Erscheinungsweise: zweimal monatlich

Abonnement unter: <http://www.deutsches-polen-institut.de/newsletter/polen-analysen/>

Auch als App für Android ${ }^{\mathrm{TM}}$ (ab Januar 2016) kostenlos auf Google Play ${ }^{\mathrm{TM}}$.

\section{Russland-Analysen}

Erscheinungsweise: zweiwöchentlich

Abonnement unter: <http://www.laender-analysen.de/russland/>

Auch als App für Android (ab Januar 2016) kostenlos auf Google Play.

\section{$D$ Google Play}

\section{Russian Analytical Digest}

In englischer Sprache. Erscheinungsweise: zweimal monatlich

Abonnement unter: <http://www.css.ethz.ch/en/publications/rad.html>

\section{Ukraine-Analysen}

Erscheinungsweise: zweimal monatlich

Abonnement unter: <http://www.laender-analysen.de/ukraine/>

Auch als App für Android (ab Januar 2016) kostenlos auf Google Play.

\section{Zentralasien-Analysen}

Erscheinungsweise: monatlich

Abonnement unter: <http://www.laender-analysen.de/zentralasien/>

Auch als App für Android (ab Januar 2016) kostenlos auf Google Play. 